\title{
VIBRATION TREATMENT MODULATES MACROPHAGE POLARISATION AND ENHANCES EARLY INFLAMMATORY RESPONSE IN OESTROGEN-DEFICIENT OSTEOPOROTIC-FRACTURE HEALING
}

\author{
S.K-H. Chow ${ }^{1,3, \$, *}$, Y-N. Chim ${ }^{1, \$}$, J. Wang ${ }^{1}$, N. Zhang ${ }^{1}$, R.M-Y. Wong ${ }^{2}$, N. Tang ${ }^{2}$, K-S. Leung ${ }^{1}$ \\ and $\mathrm{W}-\mathrm{H}$. Cheung ${ }^{1,3}$ \\ ${ }^{1}$ Musculoskeletal Research Laboratory, Department of Orthopaedics and Traumatology, \\ The Chinese University of Hong Kong, Hong Kong SAR, PR China \\ ${ }^{2}$ Department of Orthopaedics and Traumatology, Prince of Wales Hospital, Hospital Authority, \\ Hong Kong SAR, PR China \\ ${ }^{3}$ The CUHK-ACC Space Medicine Centre on Health Maintenance of Musculoskeletal System, The Chinese \\ University of Hong Kong Shenzhen Research Institute, Shenzhen, PR China \\ $\S$ Those authors contributed equally
}

\begin{abstract}
Fracture healing is a well-orchestrated and coordinated process and begins with the inflammatory stage involving the infiltration of immune cells and the release of cytokines, including tumour necrosis factor-alpha (TNF- $\alpha$ ), interleukin-6 (IL-6) and interleukin-10 (IL-10). Low-magnitude high-frequency vibration (LMHFV) stimulation is effective in promoting fracture healing. The study hypothesis was that the innate immune response was impaired in osteoporotic fracture and LMHFV could positively modulate it.

9-month-old ovariectomy (OVX)-induced osteoporotic rats were randomised into sham (SHAM), OVX control (OVX), OVX-vibration (OVX-VT) or OVX vibration plus administration of COX-2 specific non-steroid anti-inflammatory drugs (OVX-VT-NSAID). LMHFV $(35 \mathrm{~Hz}, 0.3 \times g)$ was given $20 \mathrm{~min} / \mathrm{d}$ and $5 \mathrm{~d} /$ week to the treatment groups. Healing and innate immune response were evaluated by weekly radiographs, endpoint micro-computed tomography $(\mu \mathrm{CT})$, enzyme-linked immunosorbent assay (ELISA) and histomorphometry at weeks 1,2, 4 and 8 post-treatment.

Results showed that OVX slightly elevated systemic inflammation but impaired the innate immune response locally at the fracture site, with significantly lower expressions of TNF- $\alpha$ and IL-6 but higher IL-10 expression during the early stage of healing. LMHFV was effective in accelerating the delayed fracture healing in OVX bones by partly restoring the impaired innate immune response at the fracture site, accompanied by promoted progression of macrophage polarisation from M1 (pro-inflammatory) to M2 (anti-inflammatory) phenotype. In conclusion, vibration treatment could positively modulate the impaired innate immune response and promote macrophage polarisation in osteoporotic-fracture healing.
\end{abstract}

Keywords: Osteoporotic-fracture healing, innate immunity, vibration treatment, macrophage polarisation.

*Address for correspondence: Simon Kwoon-Ho Chow, PhD, Department of Orthopaedics and Traumatology, 5/F, Clinical Sciences Building, The Chinese University of Hong Kong, Shatin, New Territories, Hong Kong SAR, PR China.

Telephone number: +852 35053312 Fax number: +852 35054618 Email: skhchow@ort.cuhk.edu.hk

Copyright policy: This article is distributed in accordance with Creative Commons Attribution Licence (http://creativecommons.org/licenses/by-sa/4.0/).

\begin{tabular}{llll}
\hline & List of Abbreviations & BV/TV & bone volume fraction \\
& callus area \\
3D & three dimensional & COX-2 & cyclooxygenase-2 \\
ckt & protein kinase B & CW & dentin matth \\
ANOVA & analysi of variance & DMP1 & phosphoprotein 1 \\
BMD & bone mineral density & & extracellular matrix \\
BV & total bone volume & ECM
\end{tabular}




\begin{tabular}{|c|c|}
\hline EDTA & $\begin{array}{l}\text { ethylenediaminetetraacetic } \\
\text { acid }\end{array}$ \\
\hline ELISA & $\begin{array}{l}\text { enzyme-linked } \\
\text { immunosorbent assay }\end{array}$ \\
\hline FACS & $\begin{array}{l}\text { fluorescence-activated cel } \\
\text { sorting }\end{array}$ \\
\hline IHC & immunohistochemistry \\
\hline IL-1 & interleukin-1 \\
\hline iNOS & $\begin{array}{l}\text { inducible nitric oxide } \\
\text { synthase }\end{array}$ \\
\hline LMHFV & $\begin{array}{l}\text { low-magnitude high- } \\
\text { frequency vibration }\end{array}$ \\
\hline MAPK & $\begin{array}{l}\text { mitogen-activated protein } \\
\text { kinase }\end{array}$ \\
\hline$\mu \mathrm{CT}$ & $\begin{array}{l}\text { micro-computed } \\
\text { tomography }\end{array}$ \\
\hline$\mu$ FEA & micro-finite element analysis \\
\hline MSCs & mesenchymal stem cells \\
\hline$N F-\kappa B$ & nuclear factor-kappa-B \\
\hline NSAIDs & $\begin{array}{l}\text { nonsteroidal anti- } \\
\text { inflammatory drugs }\end{array}$ \\
\hline OVX & ovariectomy/ovariectomised \\
\hline OVX-VT & ovariectomised vibration \\
\hline OVX-VT-NSAID & $\begin{array}{l}\text { OVX-VT with the } \\
\text { administration of NSAID }\end{array}$ \\
\hline PI3K & phosphoinositide 3-kinases \\
\hline qPCR & $\begin{array}{l}\text { quantitative polymerase } \\
\text { chain reaction }\end{array}$ \\
\hline SD & standard deviation \\
\hline TNF- $\alpha$ & tumour necrosis factor-alpha \\
\hline TV & total tissue volume \\
\hline
\end{tabular}

\section{Introduction}

Osteoporotic fracture is an increasing public health concern due to the escalating worldwide ageing population. Osteoporosis is believed to impair fracture healing leading to increased healing time, decreased mechanical strength and, thus, increased socioeconomic burden (Cheung et al., 2016). Thus, accelerating osteoporotic-fracture healing is much desired.

The success of fracture healing relies on the wellcoordinated stages of inflammation, callus formation and remodelling. The delay in osteoporotic-fracture healing is believed to be related to multifactorial impairments in each of these stages. Recruitment of reparative MSCs (Sanghani-Kerai et al., 2017; Wei et al., 2014), neo-angiogenesis at the callus (Cheung et al., 2012; Kampschulte et al., 2016), level of endochondral ossification (Shi et al., 2010) and rate of callus remodelling (Chow et al., 2011; Chow et al., 2014) are all impaired in OVX-induced osteoporotic rat fractures. These complicated processes are triggered and coordinated by the inflammatory (or innate immune) response (Claes et al., 2012). This stage of fracture repair is known as the inflammatory stage, guided and driven by the secretion of chemokines by ruptured tissues, followed by sequential recruitment of neutrophils, monocytes and macrophages (Claes et al., 2012). These innate immune cells infiltrate the fracture site and secrete inflammatory cytokines to stimulate further recruitment of reparative cells to the fracture site. The main cytokines involved in fracture healing include the pro-inflammatory TNF- $\alpha$, IL- 1 and IL- 6 and the anti-inflammatory IL-10, each taking unique surge patterns at the very early inflammatory stage and the later callus remodelling stage (Mountziaris and Mikos, 2008). Next, morphological changes of macrophages from pro-inflammatory M1 to anti-inflammatory M2 phenotype occur (Schlundt et al., 2018; Vi et al., 2015).

Oestrogen has both immunosuppressive and pro-inflammatory effects (Straub, 2007). Both TNF- $\alpha$ and IL-6 levels are indirectly mediated by the level of oestrogen through Jun or NF- $\kappa$ B pathways, respectively (Pfeilschifter et al., 2002). Moreover, oestrogen modulates inflammatory responses through oestrogen receptors, which show different expression patterns in osteoporotic fractures (Chow et al., 2014). The local inflammatory response to injury is lowered in OVX animal models (Khan and Ansar Ahmed, 2015). However, there are other studies demonstrating that oestrogen-deficiency increases the early inflammatory response after fracture, as shown by an increased number of neutrophils and expression of pro-inflammatory cytokines, midkine and IL-6 in the fracture callus in oestrogen-deficient mice (Fischer et al., 2018; Haffner-Luntzer et al., 2017). This provides further evidence of the altered inflammatory response after injury under oestrogen deficiency. However, the early inflammatory response in oestrogen-deficient osteoporotic-fracture healing has not yet been well studied.

LMHFV is a form of non-invasive, cyclic biophysical stimulation and is able to enhance OVXinduced osteoporotic-fracture healing in terms of MSC recruitment, neo-angiogenesis at the callus, endochondral ossification and callus remodelling (Cheung et al., 2012; Chow et al., 2011; Chow et al., 2016; Shi et al., 2010). Also, low-intensity vibration enhances macrophage proliferation in vitro and promote a pro-healing macrophage phenotype (Pongkitwitoon et al., 2016). However, the effects of vibration treatment on the early inflammatory response and macrophage polarisation during fracture healing are not known.

COX-2/prostaglandin signalling pathway is essential for fracture healing (Simon et al., 2002). NSAIDs are COX-2 selective inhibitors that are commonly used to reduce pain and inflammation in orthopaedic patients. In the present study, celecoxib, a type of NSAID, was administered to the animals to examine the extent to which inflammatory response participates in the enhancement of fracture healing by LMHFV.

The study hypothesis was that a difference in the inflammatory response existed between normal and osteoporotic fractures during the early inflammatory 
stage of the healing process and that mechanical stimulation in the form of vibration could modulate this response for enhancing healing in osteoporotic bones. The first objective of the study was to examine the inflammatory response of osteoporotic-fracture healing in OVX bone as compared with agematched normal bones. The second objective was to investigate the effects of vibration treatment on the early inflammatory response in osteoporotic-fracture healing.

Osteoporotic fractures demonstrated lower TNF- $\alpha$ and IL- 6 but higher IL-10 expressions locally at the fracture site at week 1 . Furthermore, vibration treatment promoted macrophage polarisation from pro-inflammatory M1 to anti-inflammatory M2 at an earlier time-point, enhanced expressions of TNF- $\alpha$ and IL- 6 and reduced IL-10 expression at the fracture site at week 1 . These findings highlighted the enhancement effects of vibration treatment on the early inflammatory response during osteoporoticfracture healing.

\section{Materials and Methods}

\section{Animal model and interventions}

$120(n=144)$ female Sprague-Dawley rats were obtained from the Laboratory Animal Services Centre of the Chinese University of Hong Kong and the experimental protocols were approved by the University Animal Experimentation Ethics Committee (Ref: 15-158-MIS). All rats were housed at the Research Animal Laboratory (The Chinese University of Hong Kong, PR China) under a $12 \mathrm{~h}$ light-dark cycle. Free cage movement was allowed, with access to standard rat chow and tap water ad libitum.

All rats received SHAM or bilateral OVX operations at 6 months of age. Rats were anaesthetised with intraperitoneal injection of $50 \mathrm{mg} / \mathrm{kg}$ ketamine and $10 \mathrm{~g} / \mathrm{kg}$ xylazine (Alfasan International B.V., Woerden, the Netherlands) both diluted in sterile $0.9 \%$ saline solution. In brief, OVX surgery was performed by making bilateral incisions at the dorsal lower abdomen followed by removal of the ovaries. Subsequently, osteoporosis developed during the following 3 months, as previously described (Cheung et al., 2011). Rats in the SHAM group received the same surgical procedures except for the ovary excision.

To address the first study's objective, 48 ninemonth-old rats were allocated and they were randomly divided into two groups: SHAM and OVX.

For the second study's objective, 96 nine-monthold rats were randomly divided into four groups: SHAM, OVX, OVX-VT and OVX-VT-NSAID. All rats were subjected to the creation of a closed fracture on the right femoral midshaft, based on an established protocol (Chow et al., 2014; Wei et al., 2014). Briefly, a $1.2 \times 150 \mathrm{~mm}$ sterilised Kirschner wire was inserted into the medullary canal followed by fracture creation using a customised 3-point bending apparatus.

$4 \mathrm{mg} / \mathrm{kg}$ (Simon et al., 2002) celecoxib (Celebrex, Pfizer), a COX-2 selective inhibitor and commonly used NSAID for pain relief in clinical setting, was administered to the OVX-VT-NSAID group through daily oral gavage, starting from $2 \mathrm{~d}$ prior to fracture creation. OVX-VT and OVX-VT-NSAID groups received LMHFV $(35 \mathrm{~Hz}, 0.3 \times g)$ treatment starting from $2 \mathrm{~d}$ after fracture creation. The treatment was given daily for $20 \mathrm{~min} / \mathrm{d}$ and $5 \mathrm{~d} /$ week, according to a previous protocol (Chow et al., 2016; Wei et al., 2016). SHAM and OVX groups were put on the turned off vibration platform.

6 rats from each group were euthanised using an overdose of intraperitoneal injection of $200 \mathrm{mg} /$ $\mathrm{kg}$ sodium pentobarbital (Dorminal $20 \%$; Alfasan International B.V.) at weeks $1,2,4$ and 8 posttreatment and femora were harvested for $\mu \mathrm{CT}$ and IHC assessments.

\section{Radiographic analysis}

Weekly radiographs of the rat femora were taken with a cabinet X-ray system (UltraFocus DXA, Faxitron, Lincolnshire, IL, USA) to monitor the fracture healing status. CA and CW were measured on digitised lateral view images using the built-in straight line and polygon selection tools in the image analysis software Image ( $\mathrm{NIH})$. CW was defined as the maximal outer diameter of the mineralised callus minus the outer diameter of the femur; CA was calculated as the sum of the areas of the external mineralised callus (Chow et al., 2016; Wei et al., 2016).

\section{D bone morphometry and $\mu F E A$}

At euthanasia, the fractured femora were harvested and scanned with a $\mu$ CT system (VivaCT 40, Scanco Medical), covering $8.02 \mathrm{~mm}$ (422 slides) proximal and distal to the fracture line, which was defined as the region of interest (Chow et al., 2014; Chow et al. (2016) Impaired inflammatory responses after oestrogen-deficient osteoporotic fracture injury. In: 15th Biennial Conference of the International Society of Fracture Repair, Munich, Germany; Wei et al., 2014). A threshold set at 220-1000 was used to distinguish between non-mineralised and mineralised tissue (Chow et al., 2014). Tissues within this set range were evaluated and reconstructed. TV, $\mathrm{BV}, \mathrm{BV} / \mathrm{TV}$ and BMD of TV and BV were assessed. Endpoint mechanical properties (week 8) in stiffness, apparent modulus and failure load were estimated by $\mu$ FEA (FE-software version 1.13, Scanco Medical) by the axial compression test in the $\mathrm{z}$-direction with the material modulus defined at $10 \mathrm{GPa}$ using the default evaluation program, as previously reported (Cheuk et al., 2015).

\section{Bone histomorphometry}

After $\mu \mathrm{CT}$ scanning, femora were fixed in $10 \%$ neutral-buffered formalin and decalcified in $9 \%$ formic acid. Processed specimens were embedded 
in paraffin-wax and cut into $5 \mu \mathrm{m}$-thick slices along the sagittal plane of the femora. Next, sections were stained with haematoxylin-eosin (Sigma-Aldrich), to visualise the callus morphometry by histology (Chow et al., 2016; Wei et al., 2016), and Martius scarlet blue (Sigma-Aldrich), to evaluate the deposition of fibrin at the fracture site. Then, fibrin area was quantified by colour threshold in ImageJ (NIH) (Chow et al., 2014).

\section{Blood collection}

At endpoints before euthanasia, whole blood sample was extracted through cardiac puncture. Part of the blood sample was collected into $\mathrm{K}_{2}$ EDTA-coated tubes (BD Medical) and part allowed to conjugate at room temperature for $2 \mathrm{~h}$ followed by centrifugation at 1,000 $\times g$ for $20 \mathrm{~min}$ for serum isolation. Samples were stored at $-20^{\circ} \mathrm{C}$ until analysis.

\section{White blood cell count}

The unclotted blood sample was used immediately to make blood smears, which were stained with DiffQuik (Shandon Kwik-Diff, Thermo Fisher Scientific) for white blood cell count using the image analysis software ImageJ.

\section{Cytokine measurements}

TNF- $\alpha$, IL- 6 and IL-10 concentrations in serum were determined by ELISA. Commercial kits (RTA00, R6000B, R1000; Quantikine ${ }^{\circledR}$ ELISA, R\&D Systems) were utilised according to the manufacturer's instructions. Optical density of each well was determined using a microplate reader ( $\mu$ Quant ${ }^{\mathrm{TM}}$, BioTek $^{\circledR}$ Instruments, Winooski, VT, USA).

\section{IHC}

After antigen retrieval in $60{ }^{\circ} \mathrm{C}$ citrate buffer, the primary antibodies for TNF- $\alpha$ (1 : 100; ab6671; Abcam), IL-6 (1 : 200; NB600-1131; Novus Biologicals, Centennial, CO, USA), IL-10 (1 : 100; ARC0102; Invitrogen), CD68 (pan-macrophage marker; 1 : 100, bs-0649R; Bioss, Woburn, MA, USA), iNOS (M1 macrophage marker; 1 : 300; NB300-605; Novus Biologicals), CD206 (M2 macrophage marker, Mannose Receptor, 1 : 200; GTX53806; GeneTex, Irvine, CA, USA) and neutrophil (1 : 2,000; LSC348181; LSBio, Seattle, WA, USA) were applied to the sections and incubated overnight at $4^{\circ} \mathrm{C}$. For negative control, primary antibodies were substituted with an isotope control antibody (IgG; GTX35035, GeneTex, Eching, Germany). All other steps followed the manufacturer's instructions for using the Mouse and Rabbit Specific HRP/DAB IHC Detection Kit-Micropolymer (ab236466; Abcam) and all specimens were processed following identical procedures. Finally, sections were counterstained with haematoxylin and images were captured using a Leica microscope system (DMRXA2). Quantitative analysis was performed at the bony callus and compared with the negative control. Expression of each target protein was quantified by colour threshold in ImageJ (Chow et al., 2014).

\section{Statistical analysis}

Quantitative data were presented as mean \pm SD and analysed in SPSS 20.0 software (IBM). Normality of data were confirmed by Kolmogorov-Smirnov test. For the data of the SHAM and OVX groups for reaching the first objective, statistical significance was assessed by independent student's $t$-test. All other data were analysed using one-way ANOVA followed by post-hoc Bonferroni's multiple comparison tests and potential associations were evaluated with Pearson's correlations. $p \leq 0.05$ was considered statistically significant.

\section{Results}

Oestrogen deficiency elevated systemic inflammation, but impaired the early innate immune response at the fracture site

Osteoporosis was confirmed by a reduction in BMD by animal dual-energy X-ray absorptiometry (UltraFocus DXA, Faxitron,Tucson, AZ, USA) in a separate batch of 4 rats. A significant drop of $13.5 \%$ in $\operatorname{BMD}\left(267.92 \pm 14.83 \mathrm{mg} / \mathrm{cm}^{2}\right)$ was detected in the $3^{\text {rd }}$ $6^{\text {th }}$ lumbar vertebra (L3-L6) when compared to those before OVX (309.75 $\left.\pm 30.6 \mathrm{mg} / \mathrm{cm}^{2} ; p=0.049\right)$. BMD in femora also showed significant decrease after OVX $\left(177.22 \pm 5.70 \mathrm{mg} / \mathrm{cm}^{2}\right)$ by $12.9 \%$ when compared to those before OVX $\left(203.50 \pm 13.18 \mathrm{mg} / \mathrm{cm}^{2} ; p=0.011\right)$ (data are displayed as mean $\pm \mathrm{SD}$ ). To examine the inflammatory response in osteoporotic-fracture healing, SHAM and OVX rats were compared. Osteoporotic-fracture healing was reconfirmed to be impaired in the OVX group as compared to normal bone in the SHAM group in terms of CW and CA (Fig. 1a,b), $\mathrm{CCT}$ evaluation with lower BV/TV, lower callus and material BMD (Fig. 1c) and lower stiffness and estimated failure loads (Fig. 5). Locally, TNF- $\alpha$ and IL-6 positive signal intensities, shown by IHC and quantified at the fracture callus at week 1 postfracture, were weaker in the OVX as compared to the SHAM group ( $p<0.05$ for both) followed by a delayed surge at week 2. Also, a stronger IL-10 signal was detected at week 1 in the OVX group $(p=0.04)$ (Fig. 2a) followed by a decreasing trend over the healing process. The SHAM group also showed a significantly stronger IL-10 positive signal at week $4(p=0.028)$. Systemically, serum TNF- $\alpha$ level was elevated in the OVX group at week $1(p=0.003)$ and $2(p=0.000)$ post-fracture as compared to the SHAM group. Significantly lower serum IL-6 level was detected at week $8(p=0.017)$ in the OVX group than in the SHAM group and the serum level of anti-inflammatory IL10 was also significantly lower at week $2(p=0.006)$ in the OVX group (Fig. 2b). Taken together, data suggested that the induction of osteoporosis by OVX induced a slight elevation in systemic inflammation but impaired the local innate immune response to fracture injury during the early phase of the healing process. 
a

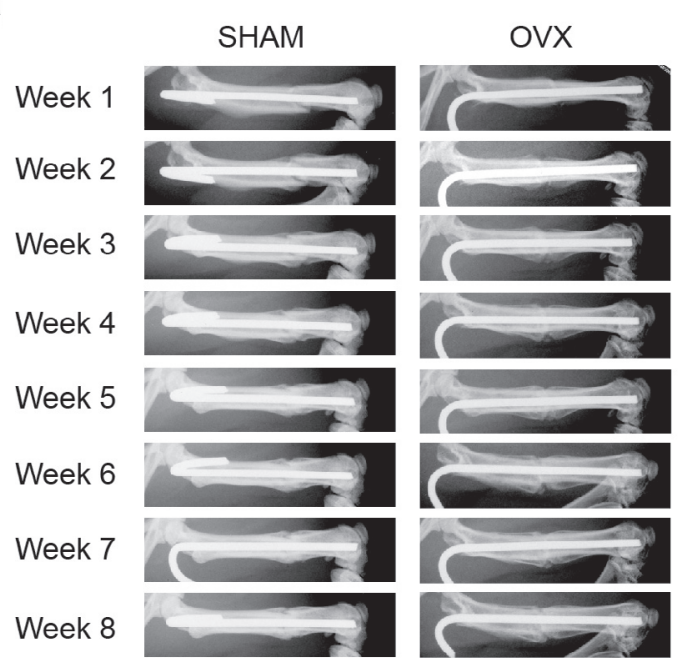

C

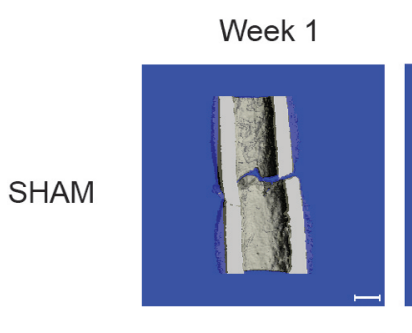

Week 1
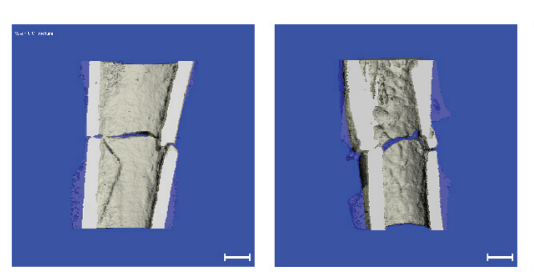

b
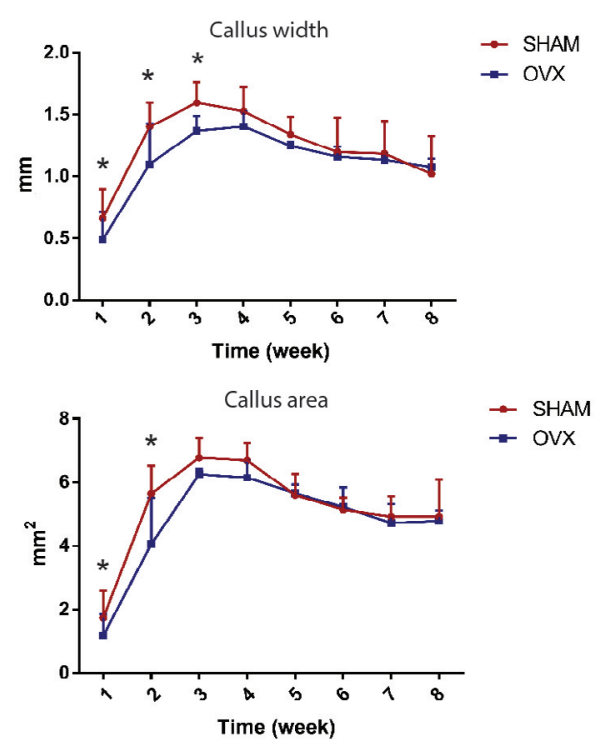

Week 4

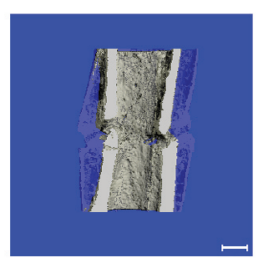

Week 8
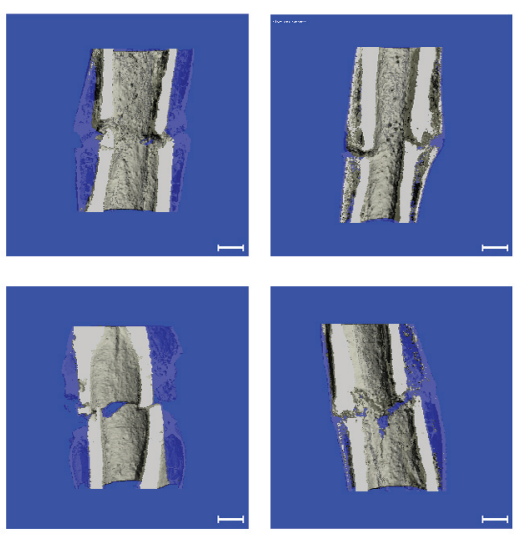
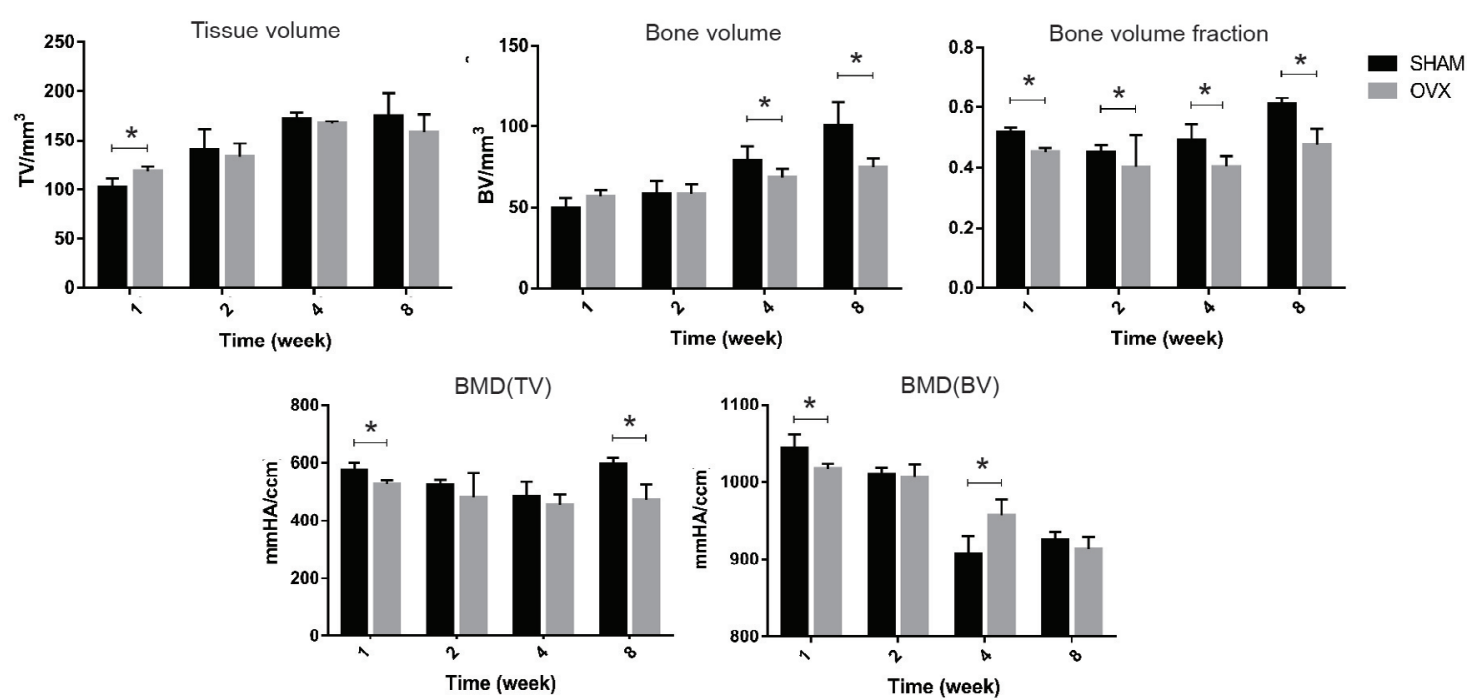

Fig. 1. Impaired healing in osteoporotic fracture. (a) Series of representative lateral radiographies of SHAM and OVX groups at different time points. Faster bridging of callus gap was observed in SHAM as compared to OVX, especially at weeks 2, 3 and 4 after fracture. (b) Quantitative analysis of temporal changes of CW and CA. OVX showed poorer callus formation as evidenced by significantly lower CW and $\mathrm{CA}$ at weeks 2 and 3 post-fracture. (c) Fractured femora were harvested and high-resolution $\mu \mathrm{CT}$ scans were performed on SHAM and OVX rats. Representative $\mu$ CT 3D reconstruction images are shown for weeks 1, 2, 4 and 8 fracture sites and callus formation. Scale bar: $1 \mathrm{~mm}$. Mean TV, BV, BV/TV, callus (TV $\mathrm{BMD}$ ) and material BMD (BV BMD) were obtained. OVX group presented inferior healing, as shown by lower BV/TV, TV BMD and BV BMD. Data are presented as mean \pm SD. Statistical analysis was performed using independent student's $t$-test. 
Vibration treatment enhanced the local innate immune response at the fracture site and fracture healing

Radiographic healing was observed to be accelerated in the OVX-VT group, with better bridging of callus gaps as compared to the OVX group, especially at week 2 and 4 (Fig. 3a), and the callus formation capacity to be significantly enhanced from week 1 to 4 post-fracture (Fig. 3b). $\mathrm{MCT}$ analysis showed that the vibration treatment enhanced the formation of a callus, as indicated by TV at week 2 . For the OVXVT-NSAID group, radiographic images and $\mu$ CT 3D images showed less newly formed callus tissue and the result was substantiated by the TV evaluation by $\mu \mathrm{CT}$ analysis. TV in the OVX-VT-NSAID group was significantly lower at week 1 and $2(p<0.05$ for all) (Fig. 3c). Higher BV/TV observed in the OVXVT-NSAID group was due to relatively lower TV across all time points. Higher callus and material BMD at week 1 could be attributed to the inferior callus formation and less newly formed bone in the OVX-VT-NSAID group. Supported by previously reported enhancement in mechanical properties (Shi et al., 2010), $\mu$ FEA analysis also showed that vibration

a

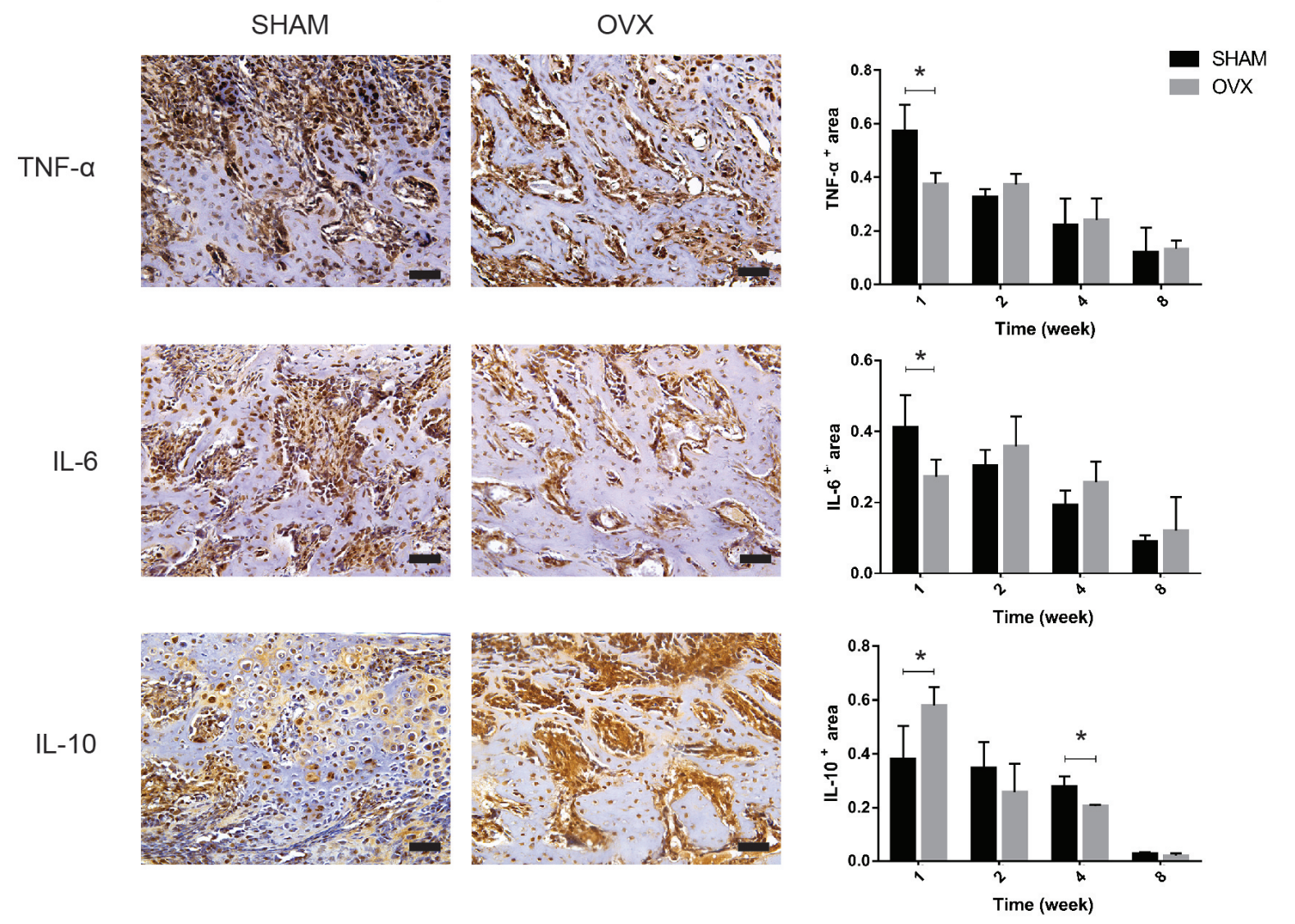

b
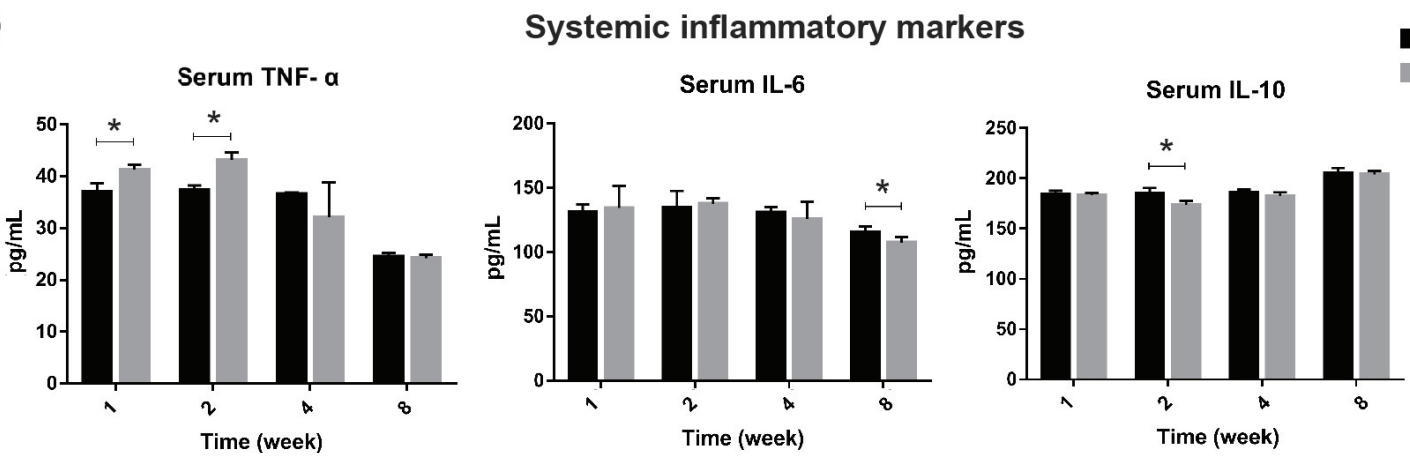

Fig. 2. Oestrogen deficiency impaired the early local innate immune response. (a) Representative TNF- $\alpha$, IL-6 and IL-10 IHC staining sections at bony callus of SHAM and OVX groups at week 1 after fracture. TNF- $\alpha$ and IL-6 were expressed at lower levels but IL-10 was expressed at higher level in OVX fractures as compared to SHAM. The corresponding area fraction of positive staining showed a locally impaired innate immune response in OVX rats as evidenced by significantly lower TNF- $\alpha$ and IL-6 expressions and higher IL-10 expression when compared to SHAM. Scale bar: $250 \mu \mathrm{m}$. (b) Serum TNF- $\alpha$, IL-6 and IL-10 levels were measured by ELISA. OVX induced a small elevation in systemic inflammation as compared to SHAM. Data are presented as mean \pm SD. Statistical analysis was performed using independent student's $t$-test. 


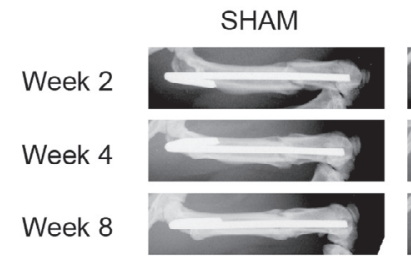

ovx

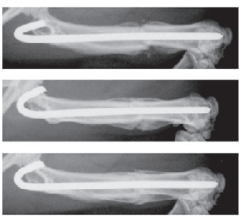

OVX-VT

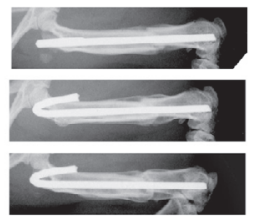

\section{Callus area}

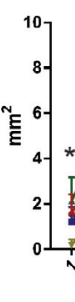

Callus area
OVX-VT-NSAID

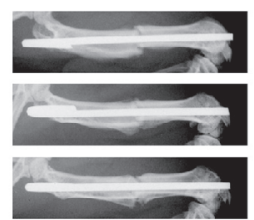

b

C

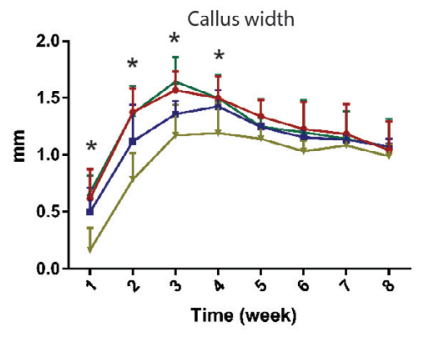

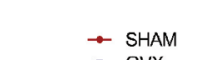 \\ $\rightarrow$ SHAM}

+ OVX-VT
+ OVX-VT-NSAID

Week 2

Week 8
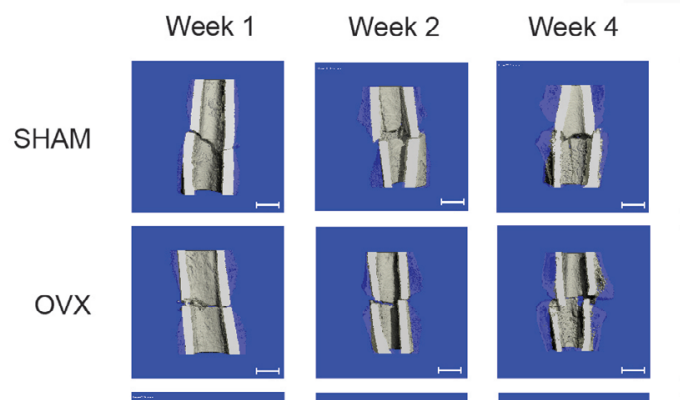

OVX-VT
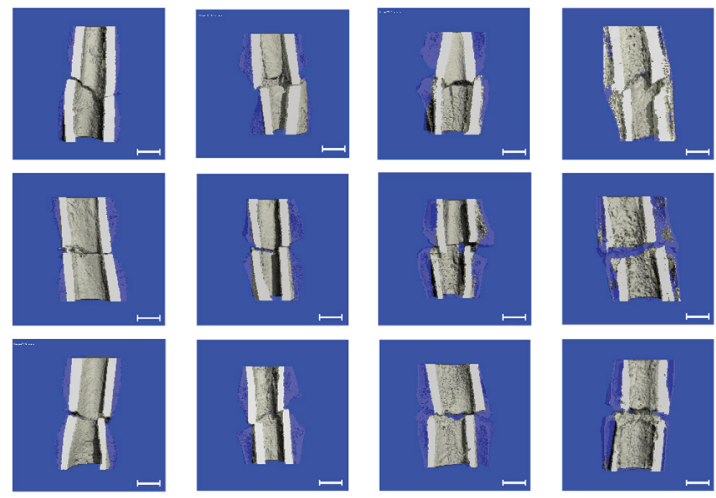

OVX-VT-NSAID
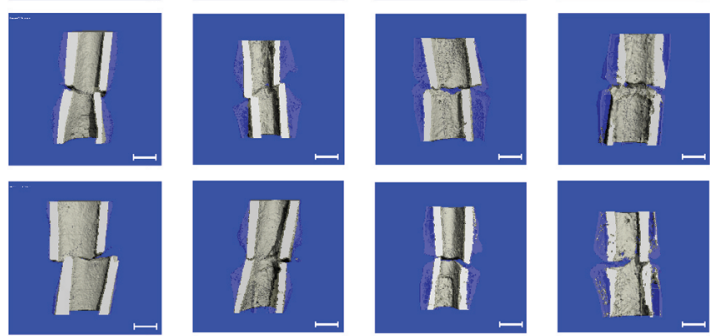

Tissue volume
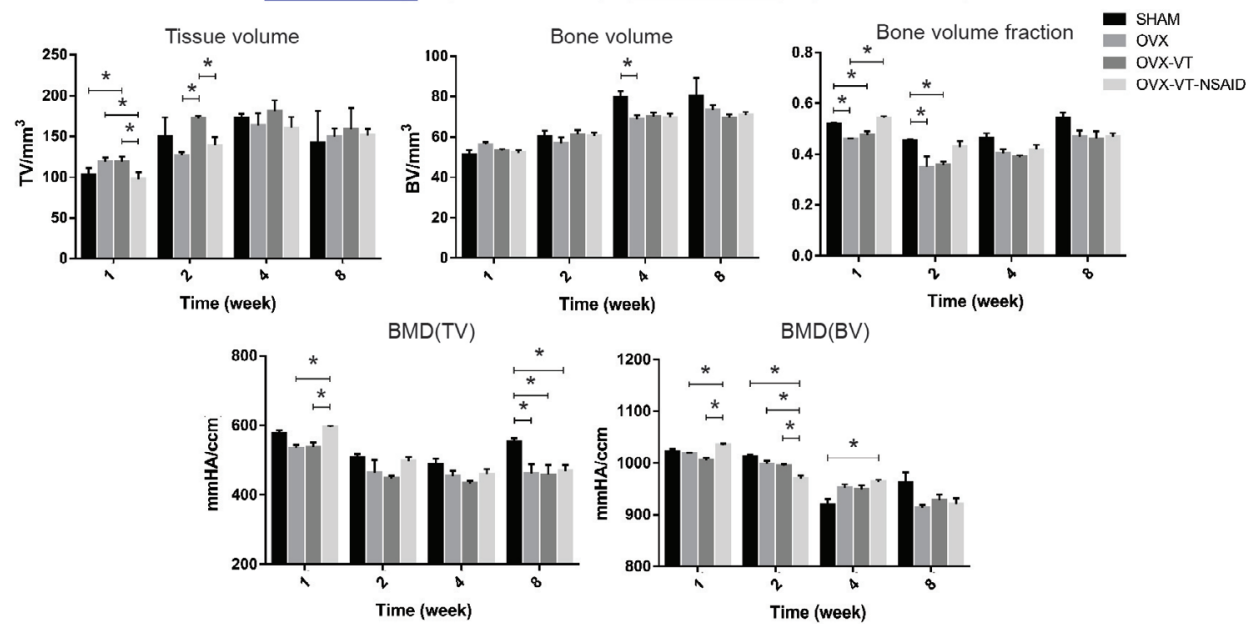

Fig. 3. Vibration accelerated healing in osteoporotic fracture while the callus formation capacity was lower with the administration of NSAID drugs. (a) Continuous radiographs of representative SHAM, OVX, OVX-VT and OVX-VT-NSAID groups at weeks 2, 4 and 8 post-fracture. More callus gaps bridging was observed in SHAM and OVX-VT when compared to OVX and OVX-VT-NSAID. (b) The temporal changes of CW and CA were quantitatively analysed. The callus formation capacity in OVX-VT was significantly enhanced from week 1 to week 4 post-fracture. OVX-VT-NSAID showed the lowest callus formation capacity across all time-points. (c) Representative $\mu \mathrm{CT} 3 \mathrm{D}$ reconstruction images of the femurs depicting the fracture sites and callus formation are shown. Scale bar: $1 \mathrm{~mm}$. OVX-VT demonstrated better bridging of callus gap at week 4 and larger callus volume as compared to OVX and OVX-VT-NSAID at week 2, also shown in TV. TV in OVX-VT-NSAID was significantly lower at weeks 1 and 2. Data are presented as mean \pm SD. Statistical analysis was performed using one-way ANOVA. 
a
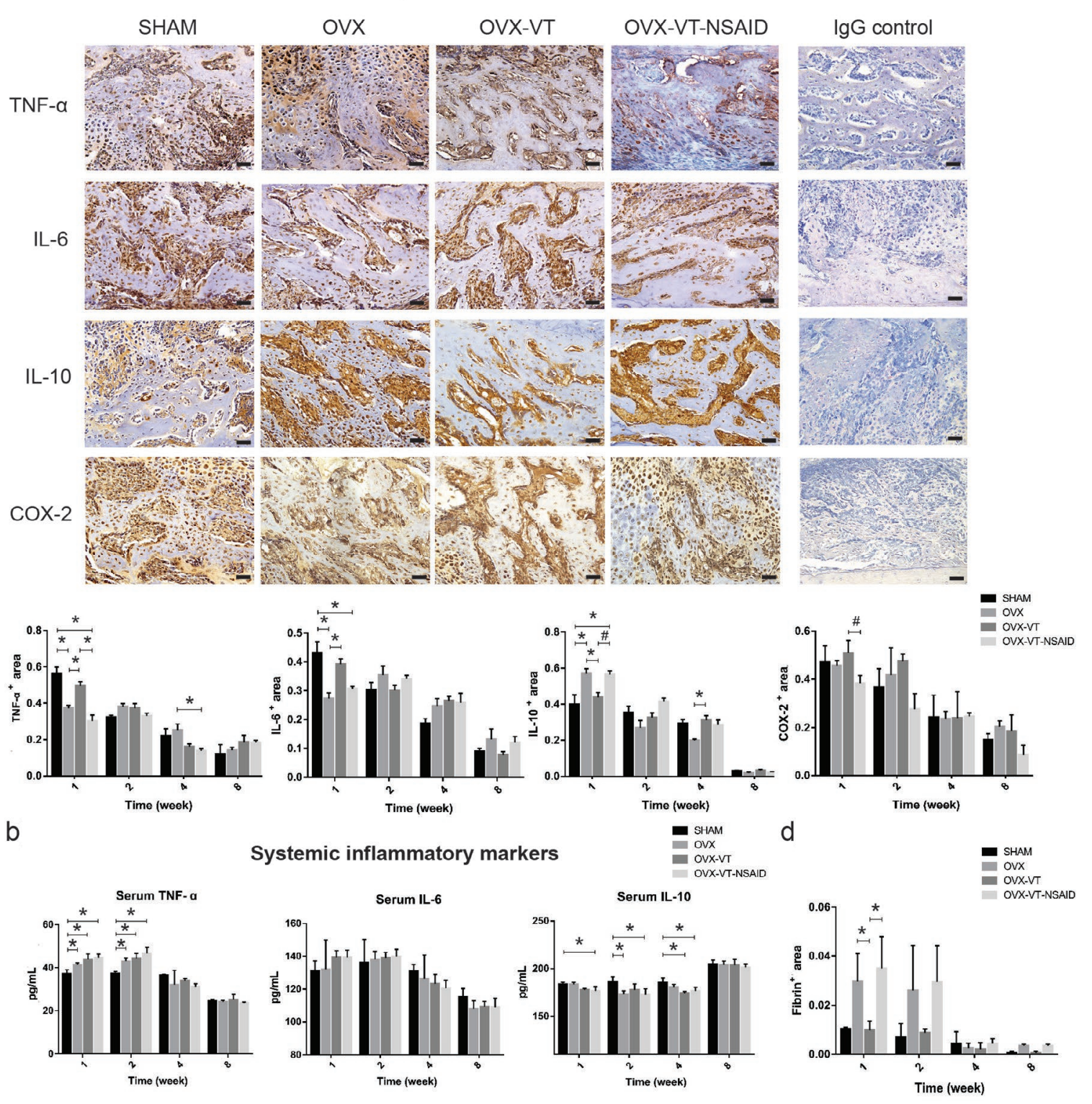

C

\section{Week 1 post-fracture}
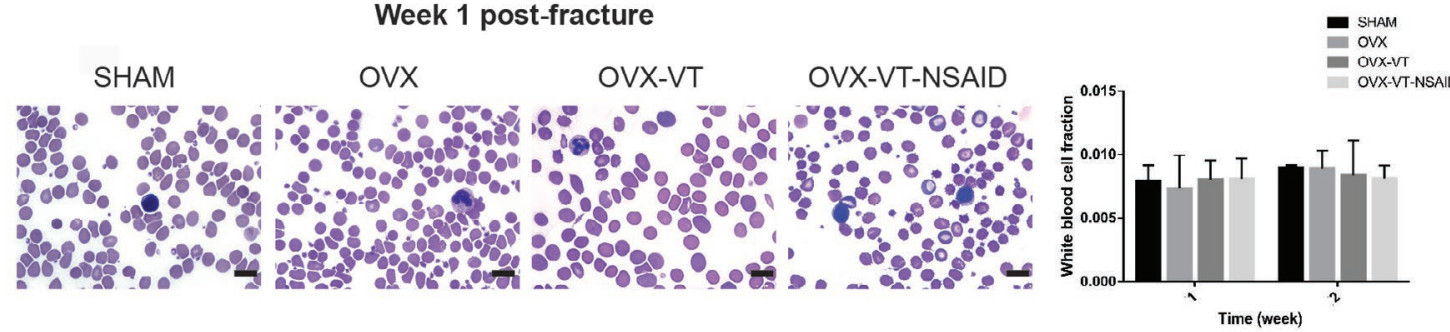

Fig. 4. At the fracture site, vibration enhanced the impaired innate immune response, which was abolished by the inhibition of COX-2. (a) Representative IHC staining sections at bony callus of SHAM, OVX, OVX-VT and OVX-VT-NSAID groups at week 1 after fracture, stained using TNF- $\alpha$, IL-6, IL-10, COX-2 and IgG control antibodies. Similarly to SHAM, OVX-VT demonstrated significantly higher TNF- $\alpha$ and IL-6 expressions but lower IL-10 expression when compared to OVX. The administration of the NSAID drug celecoxib lowered the positive effect of vibration on COX-2 expression at week 1. TNF- $\alpha$, IL-6 and IL-10 expressions at the fracture site of OVX-VT-NSAID were similar to those of OVX. Scale bar: $250 \mu \mathrm{m}$. (b) Serum TNF- $\alpha$, IL-6 and IL-10 levels were not affected by the vibration treatment. (c) Representative images of DiffQuik staining for blood smears at week 1 post-fracture and the corresponding white blood cell count at weeks 1 and 2 are shown. Systemic inflammation and inhibition of COX-2 by NSAID in terms of white blood cell count are not affected by vibration treatment. Scale bar: $375 \mu \mathrm{m}$. (d) Fibrin area was significantly lower in OVX-VT at week 1 and the trend was similar to that of SHAM. Data are presented as mean \pm SD. Statistical analysis was performed using one-way ANOVA. 
Table 1. Associations between expressions of inflammatory cytokines at the fracture callus detected by IHC against callus parameters. Statistically significant correlations were detected between TNF- $\alpha$, IL-6 and IL-10 and callus-related parameters of CW, CA, TV, BV and BMD(BV). Inflammatory cytokine expressions were correlated with callus parameters disregarding grouping and time points by Pearson correlation coefficient. ${ }^{*} p<0.05,{ }^{* *} p<0.005$.

\begin{tabular}{|c|c|c|c|c|c|c|c|}
\hline & CW & CA & TV & BV & BV/TV & BMD(TV) & BMD(BV) \\
\hline TNF- $\alpha$ & $-0.446^{* *}$ & $-0.465^{* *}$ & $-0.605^{* *}$ & $-0.649^{* *}$ & -0.082 & 0.244 & $0.703^{* *}$ \\
\hline IL-6 & $-0.458^{* *}$ & $-0.511^{* *}$ & $-0.516^{* *}$ & $-0.624^{* *}$ & -0.123 & 0.208 & $0.675^{* *}$ \\
\hline IL-10 & $-0.479^{* *}$ & $-0.555^{* *}$ & $-0.516^{* *}$ & $-0.527^{* *}$ & -0.062 & 0.223 & $0.521^{* *}$ \\
\hline
\end{tabular}
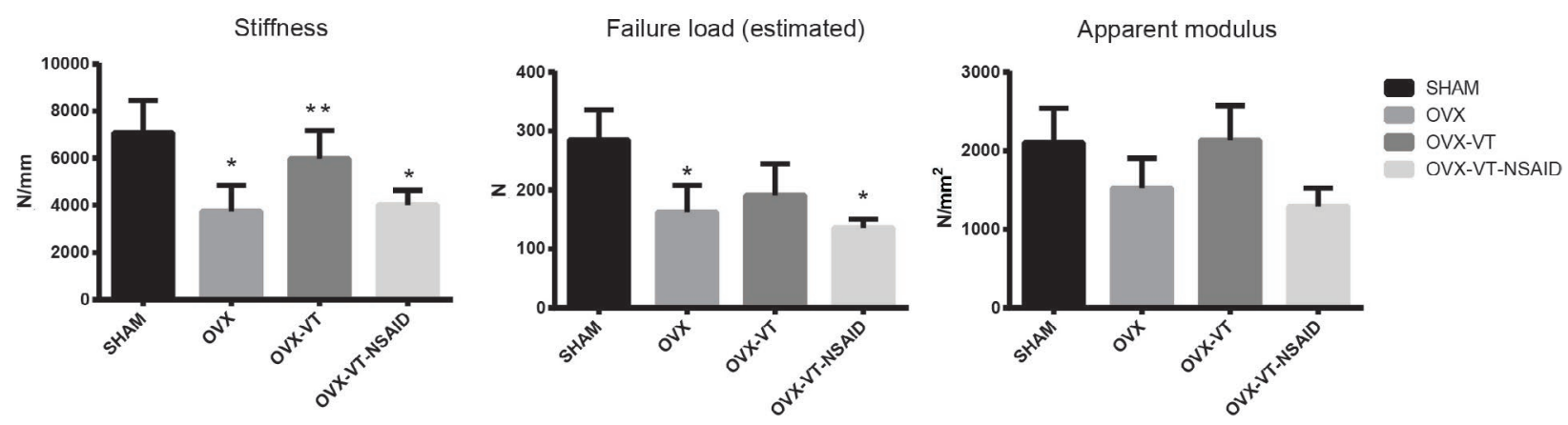

Fig. 5. $\mu$ FEA evaluation of endpoint (week 8) mechanical properties reconfirmed that the stiffness and estimated failure load were both lower in OVX and OVX-VT-NSAID groups. Stiffness enhancement by vibration treatment was also reduced by COX-2 inhibition. ${ }^{*} p<0.05$ versus SHAM, ${ }^{* *} p<0.05$ versus OVX and OVX-VT-NSAID by ANOVA with post-hoc Bonferroni test.

treatment enhanced the estimated stiffness in the OVX-VT group as compared to the OVX or OVX-VTNSAID groups (Fig. 5).

At the fracture site, the vibration treatment enhanced the signal intensities of pro-inflammatory TNF- $\alpha$ and IL- 6 and lowered the anti-inflammatory IL-10 signal as compared to the OVX group at week 1 post-fracture (Fig. 4a), showing an enhancement in the local innate immune response. The SHAM group showed a lower level of serum TNF- $\alpha$ at week 1 and 2 and a higher level of serum IL-10 at week 1, 2 and 4 . However, compared to the OVX group, the vibration treatment did not affect the systemic inflammatory status in terms of white blood cell count (Fig. 4c), as also shown by serum levels of TNF- $\alpha$, IL- 6 and IL10 across all time points (Fig. $4 \mathbf{b}$ ). At the same time, TNF- $\alpha$, IL- 6 and IL-10 positive signal intensities at the fracture site in the OVX-VT-NSAID group returned to the levels of the OVX group, indicating that the vibration-induced elevation of local innate immune response was abolished (Fig. 4a). NSAID treatment also did not show any significant influence on the systemic inflammatory status as shown by the serum levels of TNF- $\alpha$, IL-6 and IL-10 (Fig. 4b).

Moderate to strong correlations were detected between the expression of all the inflammatory cytokines at the fracture site and callus-formationrelated parameters, with statistically significant differences (Table 1). Among them, TNF- $\alpha$ and $\mathrm{BMD}(\mathrm{BV})$ showed the strongest correlation $(r=-0.703, p=0.000)$, suggesting that $49.4 \%$ of the variation in $\mathrm{BMD}(\mathrm{BV})$ could be explained by the local TNF- $\alpha$ expression.
During the first 2 weeks of fracture, IHC positive signal of COX-2 was found to be slightly elevated in the OVX-VT group as compared to the OVX group, despite not being statistically significant (Fig. 4a). However, with the suppression of the inflammatory response by celecoxib, the positive effect of vibration on COX-2 signal intensity detected at the fracture site disappeared, as shown by a marginally significantly weaker signal in the OVX-VT-NSAID group at week $1(p=0.065)$.

Fibrin deposited at the fracture site was significantly lower in the OVX-VT group as compared to the OVX and OVX-VT-NSAID groups at week 1 $(p=0.046$ and $p=0.019$, respectively) (Fig. $4 d$ ). Similar to the trend observed in the SHAM group, less fibrin was also found in the OVX-VT group at week 2 . This indicated a faster fibrin clearance by the vibration treatment at the early stage of fracture healing.

Taken together, these results suggested that vibration treatment promoted the local expression of pro-inflammatory cytokines and suppressed the expression of anti-inflammatory cytokines at the early stage of fracture healing, eventually leading to better callus formation and healing. The enhancement effect of vibration treatment was negatively modulated by the inhibition of COX-2 pathway with the administration of celecoxib.

\section{Vibration treatment accelerated macrophage} polarisation from pro- to anti-inflammatory stage The OVX-VT group demonstrated stronger signal intensity of the general macrophage marker CD68 at week 1 when compared to OVX and OVX-VT-NSAID 
groups ( $p=0.054$ for both) (Fig. 6a, b). The presence of M1 macrophages, as shown by iNOS signal, was the strongest during the early phase of fracture healing at week 1, followed by a gradual decrease and no significant difference was found between groups (Fig. 7a,b). For the detection of M2 macrophages, as shown by CD206 staining, the OVX group showed a significantly weaker signal at week $1(p=0.053)$ as compared to the OVX-VT group (Fig. 7a). At the fracture site, the OVX-VT group demonstrated a stronger CD206 positive signal at week 1 but a weaker signal at week 2 when compared to OVX and OVX-VT-NSAID groups (Fig. 7b), indicating more M2 macrophages present in the OVX-VT group at week 1 . The positive signal ratio of CD206 over iNOS (M2/M1), indicating the progress of macrophage polarisation, was further examined. In the OVXVT group, M2/M1 ratio at week 1 was significantly augmented as compared to the OVX group $(p=0.049)$, suggesting that vibration treatment had promoted the polarisation from M1 to M2 macrophages. Statistically significant negative correlations were found between CD68 and callus-related parameters of CW, CA, TV and BV $(r=-0.472, r=-0.664, r=-0.49, r=-0.469$, respectively; all $p<0.05)$, while a positive correlation was detected with $\mathrm{BMD}(\mathrm{BV})(r=0.497, p=0.014)$. A statistically significant negative correlation was also detected between M1 macrophages and CW, CA, TV and BV $(r=-0.488, r=-0.497, r=-0.574$, $r=-0.743$, respectively; all $p<0.005)$. A positive correlation was detected between M1 macrophages and BMD(TV) $(r=0.341, p<0.005)$ and BMD(BV) $(r=0.776, p<0.005)$. M2/M1 ratio showed a positive correlation with BV $(r=0.569, p<0.005)$ and BV/TV $(r=0.302, p<0.005)$ and a negative correlation with $\operatorname{BMD}(\mathrm{BV})(r=0.565, p<0.005)$ (Table 2). Furthermore, besides macrophages, the expression of neutrophils was investigated as well. The four groups had similar neutrophil signal intensities at the fracture site at all time points, with an overall decreasing trend from weeks 1 to 8 (Fig. 6a,b). This further highlighted the participation of macrophages in the enhancement of fracture healing by the vibration treatment. The observations in macrophage polarisation also suggested that the pro-inflammatory stage in osteoporotic fracture would last until week 2 post-fracture; but that the application of vibration treatment shortened the pro-inflammatory stage and promoted the shift towards the anti-inflammatory stage at an earlier time point.
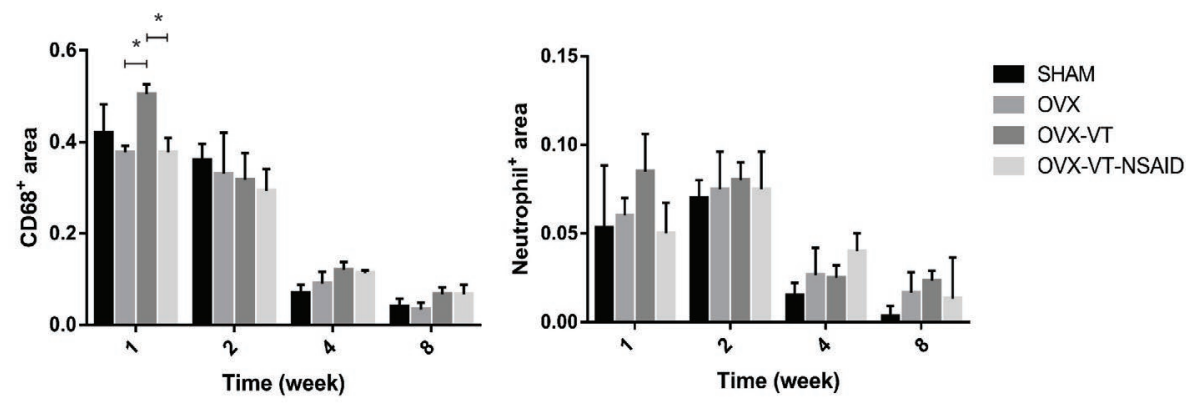

b

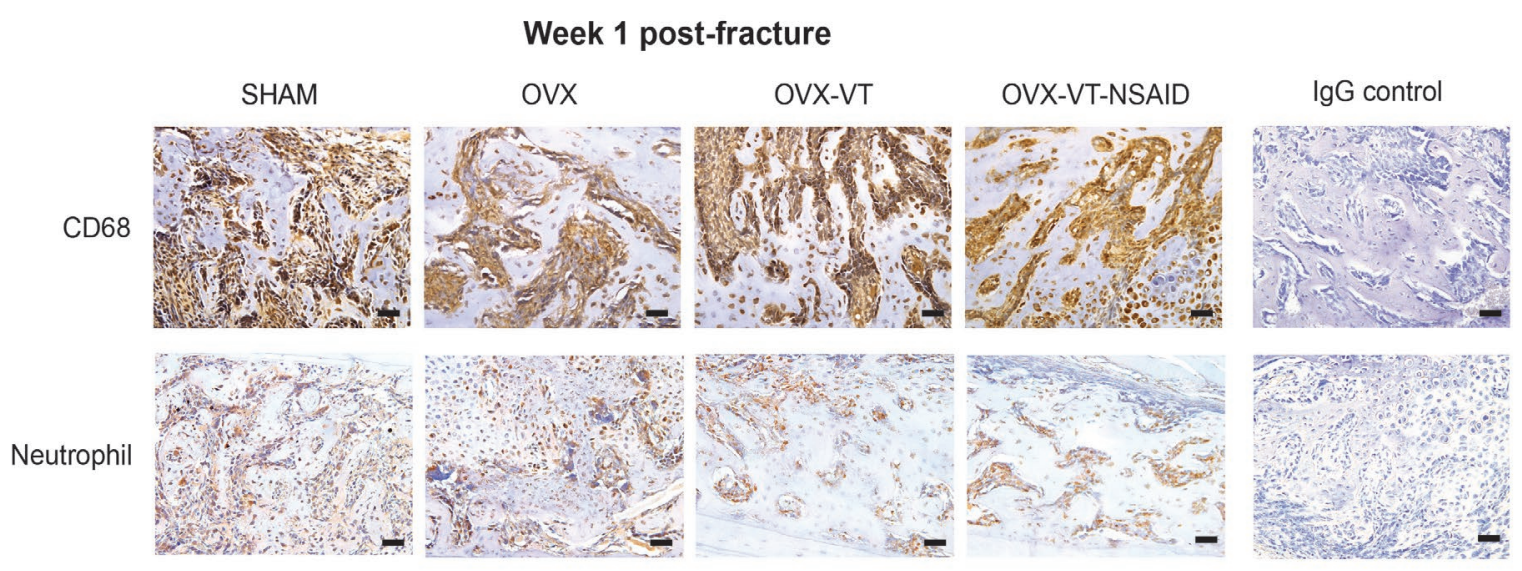

Fig. 6. Application of vibration treatment enhanced macrophage recruitment at the fracture site. (a) Area fraction of positive staining for CD68 and neutrophil. OVX-VT showed significantly higher CD68 expression as compared to OVX and OVX-VT-NSAID groups. No significant difference was detected for neutrophil between groups at all time points. Data are presented as mean \pm SD. Statistical analysis was performed using one-way ANOVA. (b) Representative IHC staining sections at bony callus of SHAM, OVX, OVX-VT and OVX-VT-NSAID groups at week 1 after fracture, stained using CD68, neutrophil and IgG control antibodies. OVX-VT demonstrated stronger CD68 positive signals at week 1 as compared to OVX and OVX-VT-NSAID. Signal intensity of neutrophils were similar between the four groups and no positive signal was detected when stained with IgG control. Scale bar: $250 \mu \mathrm{m}$. 


\section{Discussion}

The study investigated the role of vibration treatment in the innate immune response in osteoporoticfracture healing. Oestrogen depletion induced by OVX reduced the local level of inflammatory response after fracture induction as compared to normal controls. The compromised inflammatory response was enhanced by vibration treatment leading to accelerated healing, as evidenced by stronger expressions of inflammatory cytokines at the fracture site. COX-2 expression was augmented by vibration treatment in OVX rats, but this positive effect disappeared with the application of

a
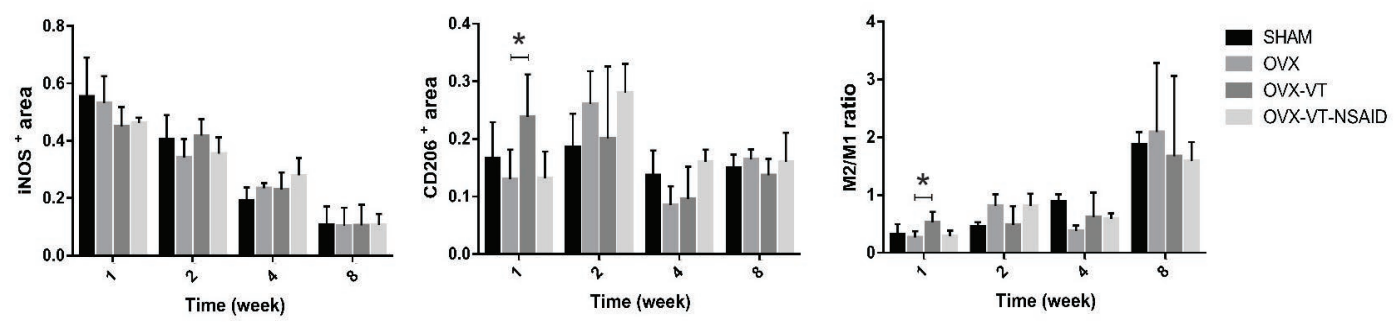

b iNOS (M1 macrophage)
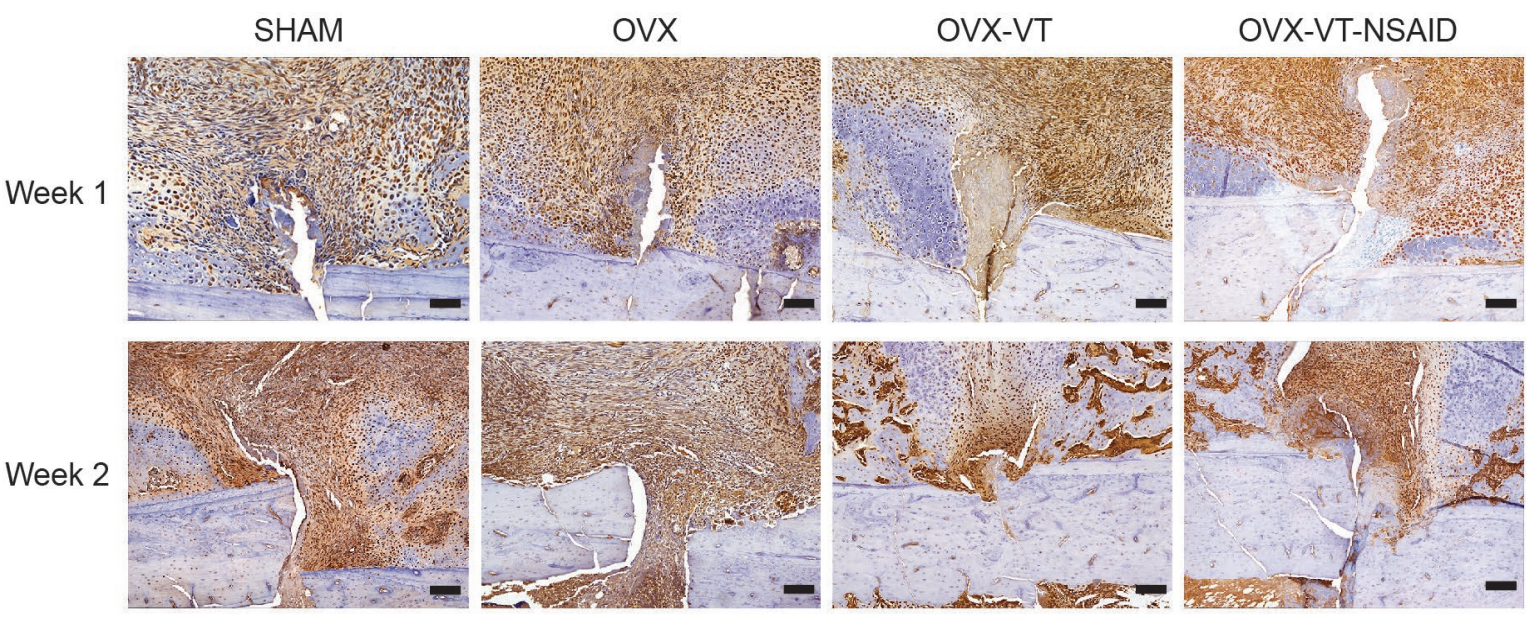

CD206 (M2 macrophage)
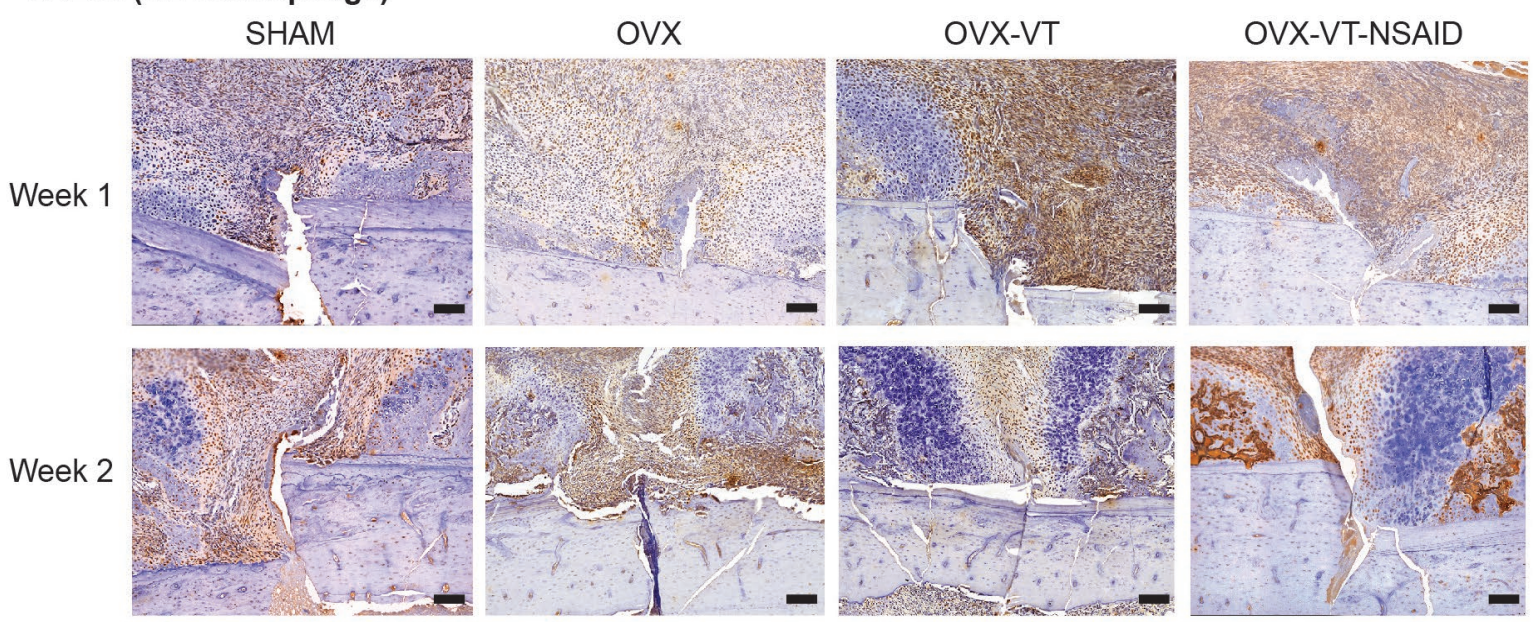

Fig. 7. Application of vibration treatment shortened the pro-inflammatory stage and promoted an earlier shift towards anti-inflammatory stage. (a) Area fraction of positive IHC staining for iNOS, CD206 and M2/ M1 ratio were analysed. iNOS expression gradually decreased from weeks 1 to 8 with no significant changes observed. OVX-VT showed significantly higher CD206 expression and M2/M1 ratio at week 1 as compared to OVX. Data are presented as mean \pm SD. Statistical analysis was performed using one-way ANOVA. (b) Representative IHC staining sections at the fracture site at weeks 1 and 2 after fracture, stained for iNOS and CD206. OVX-VT demonstrated stronger CD206 positive signals at week 1 as compared to OVX and OVX-VT-NSAID. Scale bar: $250 \mu \mathrm{m}$. 
Table 2. Associations between expression of M0, M1 and M2 macrophage markers at the fracture callus detected by IHC against callus parameters. M0 macrophage had significantly negative correlation with callus parameters of CW, CA, TV and BV and positive correlation with $B M D(B V)$. Statistically significant negative correlation was detected between M1 macrophage and CW, CA, TV and BV and positive correlation was detected against $\mathrm{BMD}(\mathrm{TV})$ and $\mathrm{BMD}(\mathrm{BV})$. M2/M1 expression ratio showed positive correlation with BV, BV/ $\mathrm{TV}$ and a negative correlation with $\mathrm{BMD}(\mathrm{BV})$. Macrophage marker expressions were correlated with callus parameters disregarding grouping and time points by Pearson correlation coefficient. ${ }^{*} p<0.05,{ }^{* *} p<0.005$.

\begin{tabular}{|c|c|c|c|c|c|c|c|}
\hline & CW & CA & TV & BV & BV/TV & BMD(TV) & BMD(BV) \\
\hline CD68 (M0) & $-0.472^{*}$ & $-0.664^{* *}$ & $-0.49^{* *}$ & $-0.469^{*}$ & 0.15 & 0.312 & $0.497^{*}$ \\
\hline iNOS (M1) & $-0.488^{* *}$ & $-0.497^{* *}$ & $-0.574^{* *}$ & $-0.743^{* *}$ & -0.043 & $0.341^{* *}$ & $0.776^{* *}$ \\
\hline CD206 (M2) & $-0.231^{*}$ & -0.201 & -0.161 & -0.167 & -0.006 & 0.132 & 0.114 \\
\hline M2/M1 ratio & 0.191 & 0.223 & 0.211 & $0.569^{* *}$ & $0.302^{* *}$ & -0.025 & $-0.565^{* *}$ \\
\hline
\end{tabular}

celecoxib, indicating that mechanical stimulation exerted a positive modulation effect on the local innate immune response leading to better healing outcomes. In addition, vibration treatment shortened the pro-inflammatory stage and promoted an earlier progression towards the anti-inflammatory stage. To the authors' knowledge, this was the first experimental study to reveal the effects of wholebody cyclic mechanical stimulation on macrophage polarisation in fracture healing.

LMHFV was proven effective in enhancing the local impaired innate immune response after fracture in OVX bone, as demonstrated by promoted local expression of pro-inflammatory cytokines and suppressed expression of anti-inflammatory cytokines, eventually leading to better callus formation and healing. In contrast to compromised fracture healing under chronic inflammatory diseases, such as rheumatoid arthritis, chronic obstructive pulmonary disease, diabetes mellitus and systemic lupus erythematosus, a specific level of local inflammatory response at the fracture site is essential for the successful coordination of the healing process. OVX-induced oestrogen depletion caused prolonged pro-inflammatory stage, lower detection of pro-inflammatory TNF- $\alpha$ and IL- 6 but higher anti-inflammatory IL-10 at the fracture site during the early phase of healing, leading to poorer healing outcomes (Fig. 2a). After fracture, inflammatory cytokines are secreted by different cell types present at the fracture site, including immune cells (e.g. neutrophils and macrophages) (Baht et al., 2018) and MSCs (Kyurkchiev et al., 2014), through the activation of different pathways, such as p38 MAPK pathway (Ono and Han, 2000). Lower inflammatory cytokine expressions in OVX rats may be partly due to inferior recruitment of immune cells to the fracture site, alterations in immune cells under oestrogen deficiency (Toniolo et al., 2015) or decreased activation of MAPK pathways, such as p38 MAPK after ovariectomy (Cong et al., 2016). Low-intensity vibration enhances macrophage proliferation in an in vitro model (Pongkitwitoon et al., 2016). The present study results are similar, as the expression of macrophages was enhanced with the application of a vibration treatment (Fig. 6a). Previous studies have also demonstrated that mechanical loading can activate the PI3K/AKT and MAPK signalling pathways and promote the production of tissue regeneration cytokines, such as IL-1 $\beta$ and IL-6, in MSCs (Fang et al., 2019) and macrophages (Bonito et al., 2019). The present study results were similar to these studies, as the expression of inflammatory cytokines was enhanced with the application of a vibration treatment (Fig. 4a). This could possibly be explained by the enhanced recruitment of macrophages to the fracture site, as shown in the present study, or the promoted activation of signalling pathways. More studies are needed to investigate the mechanism involved in this enhancement process.

Inflammatory cytokines involved in fracture healing, including TNF- $\alpha$, IL-6 and IL-10, have previously been shown to coordinate different fracture healing processes during the healing process (Mountziaris and Mikos, 2008) and the expression of these cytokines was found to correlate with callus formation (Table 1). In animal fracture models, the lack of TNF- $\alpha$ results in endochondral cartilage resorption failure (Gerstenfeld et al., 2003a) and the lack of IL-6 impairs callus maturation and delays healing (Yang et al., 2007). Enhancing the inflammatory response in fracture healing by the addition of recombinant TNF- $\alpha$ leads to better mineralisation at the fracture callus. This enhancement is abolished by the inhibition of the inflammatory response by antiTNF- $\alpha$ and various neutrophil-recruitment factors (Chan et al., 2015). Moreover, low production of IL-10 by $B$ cells is associated with delayed fracture healing (Sun et al., 2017) and B cells that produce IL-10 exert potent suppressive and regulatory functions during inflammation (Rosser et al., 2014), highlighting the importance of IL-10. Besides, a prolonged proinflammatory reaction influences negatively bone healing (Lienau et al., 2009). The present study demonstrated that vibration treatment could enhance this impaired innate immune response in OVX bone. Results showed an earlier shift from the proinflammatory stage towards the anti-inflammatory stage, demonstrated by macrophage polarisation (Fig. 7), higher expressions of pro-inflammatory cytokines and suppressed anti-inflammatory cytokine expression at the fracture site in the vibration-treated 
rats (Fig. 4a). Vibration group also showed accelerated healing of osteoporotic fracture, as evidenced by promoted callus formation at the early healing stage (Fig. 3). In previous reports, the application of various NSAIDs has been shown to delay fracture healing in rats (Beck et al., 2003; Gerstenfeld et al., 2003b). Similarly, the results of the present study showed that the enhancement by vibration treatment in OVX bone was abolished with the administration of celecoxib, a NSAID drug (Fig. 4a). Furthermore, failure to clear fibrin from the fracture site severely impaired fracture vascularisation and hindered bone union (Yuasa et al., 2015). The present study revealed that vibration treatment could accelerate the clearance of fibrin in osteoporotic bone at week 1 post-fracture, which helped enhancing fracture repair by timely and more effectively removing fibrin from the fracture site (Fig. 4d).

Macrophage expression and their polarisation from M1 to M2 phenotype were enhanced by vibration treatment. M0 macrophage marker, CD68, and M2 macrophage marker, CD206, positive signals and M2/ M1 ratio were significantly increased in OVX rats at week 1 (Fig. 6a, 7a). Indeed, the level of inflammatory response can also be evaluated by the presence of innate immune cells, including neutrophils (Kovtun et al., 2016), lymphocytes (Haffner-Luntzer et al., 2017), macrophages (Schlundt et al., 2018) and $T$ and B cells (Konnecke et al., 2014). These cells are present at the fracture site at various levels following a specific sequential order during the healing process. In particular, macrophages play a critical role in bone formation. For example, they are essential for osteoblast differentiation and, hence, healing during the callus formation stage in knockout mice models (Vi et al., 2015). The polarisation of pro-inflammatory macrophages M1 to anti-inflammatory macrophages M2 is indicative of endochondral ossification in the long bone fracture model (Cordova et al., 2017; Loi et al., 2016; Schlundt et al., 2018). In OVX mice, M1 polarisation is enhanced while M2 polarisation is impaired, leading to increased M1/M2 ratio in the bone marrow (Dou et al., 2018). The present study results revealed that the vibration treatment brought the number of M2 macrophages to normal levels, as shown by similar CD206 level to SHAM, and induced higher CD206 expression as compared to OVX rats (Fig. 7a). Also, vibration treatment shifted the increase in $\mathrm{M} 2 / \mathrm{M} 1$ ratio to week 1 instead of week 2 , indicative of possible enhancement effects in macrophage polarisation at the callus due to vibration treatment. M2 macrophages inhibit osteoclast formation (Abdelmagid et al., 2015) and support bone deposition (Xu et al., 2019). Enhancing M2 macrophages in the fracture area by the application of IL-4 and IL-13 significantly increases bone formation in a mouse osteotomy model (Schlundt et al., 2018). Macrophage morphology and activation are modulated by mechanical stimuli (McWhorter et al., 2015) and macrophages, in turn, cross-talk with mesenchymal progenitor cells to enhance osteogenesis (Pajarinen et al., 2018; Vi et al., 2015). In a mouse model, mechanical stretch induces alternative activation of macrophages (M2) to facilitate hair regeneration (Chu et al., 2019). The application of cyclic strain increases the ratio of M2/M1 macrophages in electrospun scaffolds (Ballotta et al., 2014). The current results may be the first ones demonstrating a similar phenomenon in fracture healing. Observations of macrophage phenotype in terms of $\mathrm{M} 2 / \mathrm{M} 1$ ratio and moderate-to-strong correlations with the callus size and BMD measurements showed that the M1M2 polarisation was a key inflection point when fibrin clot degradation was completed and hard callus formation was active (Table 2). Collectively, results suggested that macrophage polarisation in ovariectomised rats was prolonged as compared to normal fracture and vibration treatment could shorten this process by modulation of macrophage polarisation from M1 to M2 at an earlier time-point.

Many studies have reported the importance of COX-2/prostaglandin signalling pathway in fracture healing and that reduced expression of this pathway would hinder healing (Brown et al., 2004; Gerstenfeld and Einhorn, 2004; Naik et al., 2009; Simon et al., 2002; Xie et al., 2009; Yukata et al., 2018). In agreement with these studies, COX-2 expression was lower at the fracture site in OVX rats and healing impaired, as confirmed by inferior callus formation. At the same time, vibration treatment could positively modulate local COX-2 expression (Fig. 4a). In vitro, vibration treatment (at various frequencies) can increase substantially the expression of COX-2 in osteocytes (Lau et al., 2010) and the introduction of stretching to fibroblast can reduce the production of COX-2 (Yang et al., 2005). Similarly, in the present in vivo fracture model, vibration treatment increased COX-2 expression at the fracture callus along with other inflammatory cytokines. COX-2 is critical for regulating subsequent stages of the healing process, including chondrogenesis, callus formation and remodelling (Naik et al., 2009; Xie et al., 2008), and stimulating the differentiation of mesenchymal progenitor cells (Huang et al., 2014). The inhibition of COX-2 by a COX-2-selective inhibitor would almost completely remove the enhancement effects on all outcome measures, leading to poorer healing, as demonstrated in the present study.

The present study once again confirmed that OVXinduced osteoporotic fracture impaired inflammatory response at the early phase of fracture healing, as shown by a slight elevation in systemic inflammation, but impaired the local innate immune response. At the fracture site, the levels of pro-inflammatory markers TNF- $\alpha$ and IL- 6 were lower, whereas the level of the anti-inflammatory marker IL-10 was higher as compared to normal bones, eventually leading to compromised healing outcomes (Fig. 2a). Results were complementary to other studies showing impairment of inflammatory response in osteoporotic fracture (Wang et al., 2018) but in in contrast to Haffner-Luntzer et al. (2017), reporting 
that oestrogen deficiency elevates local neutrophils counts and expression of pro-inflammatory midkine and IL-6. Despite this, fracture healing was inevitably found to be impaired under oestrogen-deficient conditions. Due to the complex and multifaceted role of oestrogen in the immune response (Weitzmann and Pacifici, 2006), further experiments are required to delineate the role of oestrogen and demonstrate that its deficiency is certainly playing a negative role in fracture healing.

A limitation of the study was the absence of other assessments, such as qPCR or FACS. Moreover, only M1 and M2 macrophages and their interactions with mechanical stimulation in osteoporotic-fracture healing were evaluated. Thus, a more in-depth examination of various immune cell populations and their regulatory roles should be explored in future studies. Further in vitro studies will be required to delineate the effect of vibration treatment on signal transduction pathways, macrophage polarisation and the subsequent impact on osteoporotic bone regeneration. In addition, the callus quality in terms of mineralisation and collagen fibre orientation should be analysed using second-harmonic imaging microscopy, electron microscopy or nano-indentation to provide more information about callus formation.

\section{Conclusion}

The study confirmed that vibration treatment could modulate macrophage polarisation from pro-inflammatory M1 to anti-inflammatory M2 at an earlier time-point and stimulate the impaired inflammatory response in OVX bone, which exhibited decreased expressions of pro-inflammatory cytokines and increased anti-inflammatory cytokines. The findings helped to identify possible inflammationrelated therapeutic targets to tackle delayed healing or non-union in osteoporotic patients.

\section{Acknowledgements}

The study was supported by OTC Research Funds (2015-SCNT), National Natural Science Foundation of China (NSFC, 81472097) and Theme-based Research Scheme (TRS, T13-402/17-N).

\section{References}

Abdelmagid SM, Barbe MF, Safadi FF (2015) Role of inflammation in the aging bones. Life Sci 123: 25-34.

Baht GS, Vi L, Alman BA (2018) The role of the immune cells in fracture healing. Curr Osteoporos Rep 16: 138-145.

Ballotta V, Driessen-Mol A, Bouten CV, Baaijens FP (2014) Strain-dependent modulation of macrophage polarization within scaffolds. Biomaterials 35: 49194928.
Beck A, Krischak G, Sorg T, Augat P, Farker K, Merkel U, Kinzl L, Claes L (2003) Influence of diclofenac (group of nonsteroidal anti-inflammatory drugs) on fracture healing. Arch Orthop Trauma Surg 123: 327-332.

Bonito V, de Kort BJ, Bouten CVC, Smits A (2019) Cyclic strain affects macrophage cytokine secretion and extracellular matrix turnover in electrospun scaffolds. Tissue Eng Part A. DOI: 10.1089/ten. TEA.2018.0306.

Brown KM, Saunders MM, Kirsch T, Donahue HJ, Reid JS (2004) Effect of COX-2-specific inhibition on fracture-healing in the rat femur. J Bone Joint Surg Am 86-A: 116-123.

Chan JK, Glass GE, Ersek A, Freidin A, Williams GA, Gowers K, Espirito Santo AI, Jeffery R, Otto WR, Poulsom R, Feldmann M, Rankin SM, Horwood NJ, Nanchahal J (2015) Low-dose TNF augments fracture healing in normal and osteoporotic bone by up-regulating the innate immune response. EMBO Mol Med 7: 547-561.

Cheuk KY, Zhu TY, Yu FW, Hung VW, Lee KM, Qin L, Cheng JC, Lam TP (2015) Abnormal bone mechanical and structural properties in adolescent idiopathic scoliosis: a study with finite element analysis and structural model index. Calcif Tissue Int 97: 343-352.

Cheung WH, Chow SK, Sun MH, Qin L, Leung KS (2011) Low-intensity pulsed ultrasound accelerated callus formation, angiogenesis and callus remodeling in osteoporotic fracture healing. Ultrasound Med Biol 37: 231-238.

Cheung WH, Miclau T, Chow SK, Yang FF, Alt V (2016) Fracture healing in osteoporotic bone. Injury 47 Suppl 2: S21-26.

Cheung WH, Sun MH, Zheng YP, Chu WC, Leung AH, Qin L, Wei FY, Leung KS (2012) Stimulated angiogenesis for fracture healing augmented by low-magnitude, high-frequency vibration in a rat model-evaluation of pulsed-wave doppler, 3-D power Doppler ultrasonography and $\mu \mathrm{CT}$ microangiography. Ultrasound Med Biol 38: 21202129.

Chow DH, Leung KS, Qin L, Leung AH, Cheung WH (2011) Low-magnitude high-frequency vibration (LMHFV) enhances bone remodeling in osteoporotic rat femoral fracture healing. J Orthop Res 29: 746-752.

Chow SK, Leung KS, Qin J, Guo A, Sun M, Qin L, Cheung WH (2016) Mechanical stimulation enhanced estrogen receptor expression and callus formation in diaphyseal long bone fracture healing in ovariectomy-induced osteoporotic rats. Osteoporos Int 27: 2989-3000.

Chow SK, Leung KS, Qin L, Wei F, Cheung WH (2014) Callus formation is related to the expression ratios of estrogen receptors-alpha and -beta in ovariectomy-induced osteoporotic fracture healing. Arch Orthop Trauma Surg 134: 1405-1416.

Chu SY, Chou CH, Huang HD, Yen MH, Hong HC, Chao PH, Wang YH, Chen PY, Nian SX, Chen YR, Liou LY, Liu YC, Chen HM, Lin FM, Chang YT, 
Chen CC, Lee OK (2019) Mechanical stretch induces hair regeneration through the alternative activation of macrophages. Nat Commun 10: 1524. DOI: 10.1038/ s41467-019-09402-8.

Claes L, Recknagel S, Ignatius A (2012) Fracture healing under healthy and inflammatory conditions. Nat Rev Rheumatol 8: 133-143.

Cong Q, Jia H, Biswas S, Li P, Qiu S, Deng Q, Guo X, Ma G, Ling Chau JF, Wang Y, Zhang ZL, Jiang X, Liu H, Li B (2016) p38alpha MAPK regulates lineage commitment and OPG synthesis of bone marrow stromal cells to prevent bone loss under physiological and pathological conditions. Stem Cell Reports 6: 566-578.

Cordova LA, Loi F, Lin TH, Gibon E, Pajarinen J, Nabeshima A, Lu L, Yao Z, Goodman SB (2017) CCL2, CCL5, and IGF-1 participate in the immunomodulation of osteogenesis during M1/M2 transition in vitro. J Biomed Mater Res A 105: 3069 3076.

Dou C, Ding N, Zhao C, Hou T, Kang F, Cao Z, Liu C, Bai Y, Dai Q, Ma Q, Luo F, Xu J, Dong S (2018) Estrogen deficiency-mediated $\mathrm{m} 2$ macrophage osteoclastogenesis contributes to $\mathrm{M} 1 / \mathrm{M} 2$ ratio alteration in ovariectomized osteoporotic mice. J Bone Miner Res 33: 899-908.

Fang B, Liu Y, Zheng D, Shan S, Wang C, Gao Y, Wang J, Xie Y, Zhang Y, Li Q (2019) The effects of mechanical stretch on the biological characteristics of human adipose-derived stem cells. J Cell Mol Med 23: 4244-4255.

Fischer V, Kalbitz M, Muller-Graf F, Gebhard F, Ignatius A, Liedert A, Haffner-Luntzer M (2018) Influence of menopause on inflammatory cytokines during murine and human bone fracture healing. Int J Mol Sci 19. DOI: 10.3390/ijms19072070.

Gerstenfeld LC, Cho TJ, Kon T, Aizawa T, Tsay A, Fitch J, Barnes GL, Graves DT, Einhorn TA (2003a) Impaired fracture healing in the absence of TNF-alpha signaling: the role of TNF-alpha in endochondral cartilage resorption. J Bone Miner Res 18: 1584-1592.

Gerstenfeld LC, Einhorn TA (2004) COX inhibitors and their effects on bone healing. Expert Opin Drug Saf 3: 131-136.

Gerstenfeld LC, Thiede M, Seibert K, Mielke C, Phippard D, Svagr B, Cullinane D, Einhorn TA (2003b) Differential inhibition of fracture healing by non-selective and cyclooxygenase- 2 selective nonsteroidal anti-inflammatory drugs. J Orthop Res 21: 670-675.

Haffner-Luntzer M, Fischer V, Prystaz K, Liedert A, Ignatius A (2017) The inflammatory phase of fracture healing is influenced by oestrogen status in mice. Eur J Med Res 22: 23. DOI: 10.1186/s40001-0170264-y.

Huang C, Xue M, Chen H, Jiao J, Herschman HR, O'Keefe RJ, Zhang X (2014) The spatiotemporal role of COX-2 in osteogenic and chondrogenic differentiation of periosteum-derived mesenchymal progenitors in fracture repair. PLoS One 9: e100079. DOI: 10.1371/journal.pone.0100079.

Kampschulte M, Krombach GA, Richards DC, Sender J, Lips KS, Thormann U, El Khassawna T, Ray S, Alt V, Langheinrich AC (2016) Neovascularization of osteoporotic metaphyseal bone defects: a morphometric $\mu C T$ study. Microvasc Res 105: 7-14.

Khan D, Ansar Ahmed S (2015) The immune system is a natural target for estrogen action: opposing effects of estrogen in two prototypical autoimmune diseases. Front Immunol 6: 635. DOI: 10.3389/fimmu.2015.00635.

Konnecke I, Serra A, El Khassawna T, Schlundt C, Schell H, Hauser A, Ellinghaus A, Volk HD, Radbruch A, Duda GN, Schmidt-Bleek K (2014) T and B cells participate in bone repair by infiltrating the fracture callus in a two-wave fashion. Bone 64: 155-165.

Kovtun A, Bergdolt S, Wiegner R, Radermacher P, Huber-Lang M, Ignatius A (2016) The crucial role of neutrophil granulocytes in bone fracture healing. Eur Cell Mater 32: 152-162.

Kyurkchiev D, Bochev I, Ivanova-Todorova E, Mourdjeva M, Oreshkova T, Belemezova K, Kyurkchiev S (2014) Secretion of immunoregulatory cytokines by mesenchymal stem cells. World J Stem Cells 6: 552-570.

Lau E, Al-Dujaili S, Guenther A, Liu D, Wang L, You L (2010) Effect of low-magnitude, highfrequency vibration on osteocytes in the regulation of osteoclasts. Bone 46: 1508-1515.

Lienau J, Schmidt-Bleek K, Peters A, Haschke F, Duda GN, Perka C, Bail HJ, Schutze N, Jakob F, Schell H (2009) Differential regulation of blood vessel formation between standard and delayed bone healing. J Orthop Res 27: 1133-1140.

Loi F, Cordova LA, Zhang R, Pajarinen J, Lin TH, Goodman SB, Yao Z (2016) The effects of immunomodulation by macrophage subsets on osteogenesis in vitro. Stem Cell Res Ther 7: 15. DOI: 10.1186/s13287-016-0276-5.

McWhorter FY, Davis CT, Liu WF (2015) Physical and mechanical regulation of macrophage phenotype and function. Cell Mol Life Sci 72: 1303-1316.

Mountziaris PM, Mikos AG (2008) Modulation of the inflammatory response for enhanced bone tissue regeneration. Tissue Eng Part B Rev 14: 179-186.

Naik AA, Xie C, Zuscik MJ, Kingsley P, Schwarz EM, Awad H, Guldberg R, Drissi H, Puzas JE, Boyce B, Zhang X, O'Keefe RJ (2009) Reduced COX-2 expression in aged mice is associated with impaired fracture healing. J Bone Miner Res 24: 251-264.

Ono K, Han J (2000) The p38 signal transduction pathway: activation and function. Cell Signal 12: 1-13.

Pajarinen J, Lin T, Gibon E, Kohno Y, Maruyama M, Nathan K, Lu L, Yao Z, Goodman SB (2018) Mesenchymal stem cell-macrophage crosstalk and bone healing. Biomaterials 196: 80-89.

Pfeilschifter J, Koditz R, Pfohl M, Schatz H (2002) Changes in proinflammatory cytokine activity after menopause. Endocr Rev 23: 90-119. 
Pongkitwitoon S, Weinheimer-Haus EM, Koh TJ, Judex S (2016) Low-intensity vibrations accelerate proliferation and alter macrophage phenotype in vitro. J Biomech 49: 793-796.

Rosser EC, Blair PA, Mauri C (2014) Cellular targets of regulatory B cell-mediated suppression. Mol Immunol 62: 296-304.

Sanghani-Kerai A, Coathup M, Samazideh S, Kalia P, Di Silvio L, Idowu B, Blunn G (2017) Osteoporosis and ageing affects the migration of stem cells and this is ameliorated by transfection with CXCR4. Bone Joint Res 6: 358-365.

Schlundt C, El Khassawna T, Serra A, Dienelt A, Wendler S, Schell H, van Rooijen N, Radbruch A, Lucius R, Hartmann S, Duda GN, Schmidt-Bleek K (2018) Macrophages in bone fracture healing: their essential role in endochondral ossification. Bone 106: 78-89.

Shi HF, Cheung WH, Qin L, Leung AH, Leung KS (2010) Low-magnitude high-frequency vibration treatment augments fracture healing in ovariectomyinduced osteoporotic bone. Bone 46: 1299-1305.

Simon AM, Manigrasso MB, O'Connor JP (2002) Cyclo-oxygenase 2 function is essential for bone fracture healing. J Bone Min Res 17: 963-976.

Straub RH (2007) The complex role of estrogens in inflammation. Endocr Rev 28: 521-574.

Sun GJ, Wang YC, Ti YF, Wang J, Zhao JN, Qian HB (2017) Regulatory B cell is critical in bone union process through suppressing proinflammatory cytokines and stimulating Foxp3 in Treg cells. Clin Exp Pharmacol Physiol 44: 455-462.

Toniolo A, Fadini GP, Tedesco S, Cappellari R, Vegeto E, Maggi A, Avogaro A, Bolego C, Cignarella A (2015) Alternative activation of human macrophages is rescued by estrogen treatment in vitro and impaired by menopausal status. J Clin Endocrinol Metab 100: E50-58.

Vi L, Baht GS, Whetstone H, Ng A, Wei Q, Poon R, Mylvaganam S, Grynpas M, Alman BA (2015) Macrophages promote osteoblastic differentiation in-vivo: implications in fracture repair and bone homeostasis. J Bone Min Res 30: 1090-1102.

Wang JS, He M, Wang GB, Fu Q (2018) Organic gallium treatment improves osteoporotic fracture healing through affecting the OPG/RANKL ratio and expression of serum inflammatory cytokines in ovariectomized rats. Biol Trace Elem Res 183: 270-279.

Wei FY, Chow SK, Leung KS, Qin J, Guo A, Yu OL, Li G, Cheung WH (2016) Low-magnitude highfrequency vibration enhanced mesenchymal stem cell recruitment in osteoporotic fracture healing through the SDF-1/CXCR4 pathway. Eur Cell Mater 31: 341354.

Wei FY, Leung KS, Li G, Qin J, Chow SK, Huang S, Sun MH, Qin L, Cheung WH (2014) Low intensity pulsed ultrasound enhanced mesenchymal stem cell recruitment through stromal derived factor-1 signaling in fracture healing. PLoS One 9: e106722. DOI: 10.1371/journal.pone.0106722.
Weitzmann MN, Pacifici R (2006) Estrogen deficiency and bone loss: an inflammatory tale. J Clin Invest 116: 1186-1194.

Xie C, Liang B, Xue M, Lin AS, Loiselle A, Schwarz EM, Guldberg RE, O'Keefe RJ, Zhang X (2009) Rescue of impaired fracture healing in COX-2-/- mice via activation of prostaglandin E2 receptor subtype 4 . Am J Pathol 175: 772-785.

Xie C, Ming X, Wang Q, Schwarz EM, Guldberg RE, O'Keefe RJ, Zhang X (2008) COX-2 from the injury milieu is critical for the initiation of periosteal progenitor cell mediated bone healing. Bone 43: 10751083.

Xu X, Wang R, Sun Z, Wu R, Yan W, Jiang Q, Shi $D$ (2019) Trehalose enhances bone fracture healing in a rat sleep deprivation model. Ann Transl Med 7: 297. DOI: 10.21037/atm.2019.05.73.

Yang G, Im HJ, Wang JH (2005) Repetitive mechanical stretching modulates IL-1beta induced COX-2, MMP-1 expression, and PGE2 production in human patellar tendon fibroblasts. Gene 363: 166-172.

Yang X, Ricciardi BF, Hernandez-Soria A, Shi Y, Pleshko Camacho N, Bostrom MP (2007) Callus mineralization and maturation are delayed during fracture healing in interleukin- 6 knockout mice. Bone 41: 928-936.

Yuasa M, Mignemi NA, Nyman JS, Duvall CL, Schwartz HS, Okawa A, Yoshii T, Bhattacharjee G, Zhao C, Bible JE, Obremskey WT, Flick MJ, Degen JL, Barnett JV, Cates JM, Schoenecker JG (2015) Fibrinolysis is essential for fracture repair and prevention of heterotopic ossification. J Clin Invest 125: 3117-3131.

Yukata K, Xie C, Li TF, Brown ML, Kanchiku T, Zhang X, Awad HA, Schwarz EM, Beck CA, Jonason JH, O'Keefe RJ (2018) Teriparatide (human PTH1-34) compensates for impaired fracture healing in COX-2 deficient mice. Bone 110: 150-159.

\section{Discussion with Reviewers}

Thaqif El Khassawna: Would the authors expect a deviant result if a more stable (rigid) fixation method was used?

Authors: We have previously utilised a metaphyseal fracture model with complete osteotomy at the metaphysis unilaterally and rigid plate fixation (Wong et al., 2018; Wong et al., 2019; additional references). In addition, vibration treatment enhanced early phase fibrinolysis in a metaphyseal fracture model, which helped to accelerate the overall fracture healing by timely and more effectively removing fibrin from the fracture site (study submitted). Others have utilised a mouse model subjected to vibration treatment after femur osteotomy stabilised by a semi-rigid external fixator and found that OVX-induced compromised fracture healing is significantly improved in terms of callus properties and bone formation (HaffnerLuntzer et al., 2018; Wehrle et al., 2015; additional 
references). Thus, we speculate that the results would be very similar if a more rigid fixation was used.

Thaqif El Khassawna: Do the authors expect an improved or a discrepant bone quality in terms of collagen fibre orientation and arrangement, osteocyte morphology and ECM components in response to the treatment? Are there any data in this regard?

Authors: Chung et al. (2014, additional reference) demonstrated that vibration treatment could upregulate the expression levels of collagen type I and II in the fracture callus at different fracture healing phases, resulting in enhanced callus formation and mineralisation. Moreover, mechanical loading influences ECM composition depending on loading conditions in in vitro models (Sebastine and Williams, 2006; additional reference). The ECM network is structurally remodelled when being exposed to mechanical stress and strain that change fibre orientations in the direction of loads and their connectivity (Kim et al., 2019; additional reference). Therefore, we would expect vibration-treated animals to have different collagen fibre orientation and arrangement as well as ECM components when compared with controls, leading to improved bone quality. However, data about collagen fibres orientation and arrangement and ECM components could not be provided in the present study. More in vitro experiments could be performed to verify the above hypothesis.

As for the osteocyte morphology, osteocytes of vibration-treated animals have remarkably more dendrites radiated around the cell body as compared to control ones, with significantly higher expression of DMP1 and E11 (an osteocyte marker) at dendrites (study under revision).

Thaqif El Khassawna: Would the authors expect discrepant collagen fibres orientation if they would have performed a sirius red staining and evaluated it using polarised-light microscopy?

Authors: Based on the level of mechanical stimulation received by the treated animals and the biological effect, as demonstrated by the enhanced callus formation, it is possible that vibration-treated animals would have very different collagen fibre orientation when compared with control animals. This may be associated with the direction of the vibration in relation to the bone orientation (different in animal and human). However, collagen fibre orientation data could not be provided in the present study.

Thaqif El Khassawna: Do the authors believe that vibration therapy is a viable replacement for Food and Drug Administration-approved osteoporosis prevention medications? Or is the potential higher as an enhancing approach for bone healing?

Authors: We believe that vibration therapy could be a viable approach for both osteoporosis prevention and bone healing enhancement. Vibration therapy is able to maintain BMD in human subjects, reduce fall and fracture rates (Leung et al., 2014, additional reference) and enhance fracture healing in animals (Wang et al., 2017, additional reference).

In order to apply vibration therapy in humans, more well-designed randomised controlled clinical trials could be performed to further examine the efficacy, regimes and safety of vibration treatment.

Thaqif El Khassawna: Is there a limit to the modulation of the innate immune system by means of vibration? In other words, will vibration treatment reach any harmful extent? Would the excessive polarisation of M2 pose a risk to the balanced patients' immune response? If so, what are the suggested parameters to monitor such polarisation? Authors: We believe that a limit to the modulation of the innate immune response by vibration exists. However, we did not investigate the dose-dependent effects of vibration on the inflammatory response. Thus, more studies are still needed to investigate this aspect.

A balanced inflammatory response is essential not only to successful fracture healing but also other injury healing processes. Hence, excessive polarisation of M2 is believed to disturb the balance of the immune response as a whole, but promoting the inferior level of M2 polarisation at the fracture site could help accelerate fracture healing, as shown in the present study. Vibration treatment enhanced the sub-optimal inflammatory response in oestrogendeficient osteoporotic animals, leading to more favourable healing outcomes.

As for monitoring M2 polarisation, levels of M2 markers in serum could be tested.

Melanie Haffner-Luntzer: It would be interesting to discuss the translational relevance of the vibration treatment. How much strain arises locally at the fracture callus due to the vibration and how would this transfer to the clinical situation?

Authors: Strain at the fracture callus was not measured in the present study. Dynamic strain magnitudes collected from the surface of a rat proximal tibia induced at $45 \mathrm{~Hz}$ is $2.12 \pm 0.42 \mu \varepsilon$ (Judex et al., 2007, additional reference). For clinical application, since the changes in vibration frequency may have an impact on the ability of the vibration treatment to enhance bone formation, further randomised controlled clinical trials are needed to determine the optimal regime of vibration treatment.

\section{Additional References}

Chung SL, Leung KS, Cheung WH (2014) Lowmagnitude high-frequency vibration enhances gene expression related to callus formation, mineralization and remodeling during osteoporotic fracture healing in rats. J Orthop Res 32: 1572-1579. 
Kim H, Kim MC, Asada HH (2019) Extracellular matrix remodelling induced by alternating electrical and mechanical stimulations increases the contraction of engineered skeletal muscle tissues. Sci Rep 9: 2732. DOI: 10.1038/s41598-019-39522-6.

Haffner-Luntzer M, Kovtun A, Lackner I, Modinger Y, Hacker S, Liedert A, Tuckermann J, Ignatius A (2018) Estrogen receptor alpha- (ERalpha), but not ERbeta-signaling, is crucially involved in mechanostimulation of bone fracture healing by whole-body vibration. Bone 110: 11-20.

Judex S, Lei X, Han D, Rubin C (2007) Lowmagnitude mechanical signals that stimulate bone formation in the ovariectomized rat are dependent on the applied frequency but not on the strain magnitude. J Biomech 40: 1333-1339.

Leung KS, Li CY, Tse YK, Choy TK, Leung PC, Hung VW, Chan SY, Leung AH, Cheung WH (2014) Effects of 18-month low-magnitude high-frequency vibration on fall rate and fracture risks in 710 community elderly - a cluster-randomized controlled trial. Osteoporos Int 25: 1785-1795.

Sebastine IM, Williams DJ (2006) The role of mechanical stimulation in engineering of extracellular matrix (ECM). Conf Proc IEEE Eng Med Biol Soc 1: 3648-3651.

Wang J, Leung KS, Chow SK, Cheung WH (2017) The effect of whole body vibration on fracture healing - a systematic review. Eur Cell Mater 34: 108-127.

Wehrle E, Liedert A, Heilmann A, Wehner T, Bindl R, Fischer L, Haffner-Luntzer M, Jakob F, Schinke T, Amling M, Ignatius A (2015) The impact of lowmagnitude high-frequency vibration on fracture healing is profoundly influenced by the oestrogen status in mice. Dis Model Mech 8: 93-104.

Wong RMY, Choy MHV, Li MCM, Leung KS, K-H Chow S, Cheung WH, Cheng JCY (2018) A systematic review of current osteoporotic metaphyseal fracture animal models. Bone Joint Res 7: 6-11.

Wong RM, Thormann U, Choy MH, Chim YN, Li MC, Wang JY, Leung KS, Cheng JC, Alt V, Chow SK, Cheung WH (2019) A metaphyseal fracture rat model for mechanistic studies of osteoporotic bone healing. Eur Cell Mater 37: 420-430.

Editor's note: The Scientific Editor responsible for this paper was Juerg Gasser. 